\title{
Title: Velocity controlled sound field reproduction by non-uniformly spaced loudspeakers
}

Running Title: Velocity controlled sound field reproduction

\author{
Mincheol Shin* a), Philip A. Nelson ${ }^{\text {a) }}$, Filippo M. Fazi ${ }^{\text {a) }}$ and Jeongil Seo ${ }^{\text {b) }}$
}

a) Institute of Sound and Vibration Research (ISVR), University of Southampton, University Road, Highfield, Southampton S017 1BJ, United Kingdom

b) Department of Broadcasting and Telecommunications Media Research, Electronics Telecommunication Research Institute (ETRI),

138 Gajeongno, Yuseong-gu, Daejeon, 305-700, Republic of Korea

* Electronic mail of corresponding author: M.Shin@soton.ac.uk

Submitted to Journal of Sound and Vibration (JSV): February, 2015

First revision: September, 2015

Second revision: December, 2015 


\begin{abstract}
One approach to the reproduction of a sound field is to ensure the reproduction of the acoustic pressure on the surface bounding the volume within which reproduction is sought. However, this approach suffers from technical limitations when the loudspeakers used for the reproduction of the surface acoustic pressures are unevenly spaced. It is shown in this paper that sound field reproduction with a spatially non-uniform loudspeaker arrangement can be considerably improved by changing the physical quantity to be controlled on the bounding surface from pressure to particle velocity. One of the main advantages of the velocity control method is the simplicity with which the inverse problem can be regularized, irrespective of the direction of arrival of the sound to be reproduced. In addition, the velocity controlled sound field shows better reproduction of the time averaged intensity flow in the reproduction region which in turn appears to be closely linked with better human perception of sound localization. Furthermore, the proposed method results in smoother "panning functions" that describe the variation of the source outputs as a function of the angle of incidence of the sound to be reproduced. The performance of the velocity matching method has been evaluated by comparison to the conventional pressure matching method and through simulations with several non-uniform loudspeaker layouts. The simulated results were also verified with experiments and subjective tests.
\end{abstract}




\section{INTRODUCTION}

Due to recently emerging interests in the production of virtual three-dimensional (3D) experiences, technologies for reproducing virtual 3D scenes have become one of the most important issues in the fields of entertainment, broadcasting and multi-media content. The rapid development of visual 3D technologies widely popularized in cinema and home theatre has led to the requirement for better aural $3 \mathrm{D}$ technologies in order to deliver immersive auditory experiences to consumers. A new generation of broadcasting service equipped with 3D spatial audio technology is fast becoming a real possibility.

Binaural technology has proven to be an efficient and effective way for reproducing a 3D sound scene using relatively small numbers of loudspeakers with the clear limitation that a single binaural reproduction system cannot replicate the 3D sound image for multiple listeners over a wide area [1,2]. Several technical methods using multiple loudspeakers have been proposed for generating spatial audio experience for more people in a wider area. Wave Field Synthesis (WFS), which produces artificial wave fronts synthesized by multiple loudspeakers based on the Kirchhoff-Helmholtz integral, was developed in order to obtain the signal inputs to an array of multiple sound sources to reproduce the virtual sound field $[3,4]$. Ambisonics, which is another method used in multichannel audio (also referred to as a fullsphere surround sound technique), is based on a spherical harmonic analysis of the field to be reproduced, the spherical harmonics providing a means of describing a 3D sound field in terms of natural spatial basis functions $[5,6]$. The pressure matching technique using a least squares method $[7,8]$ has also been applied to the problem of sound field reproduction. This method delivers the source inputs ensuring the reproduction of the target acoustic pressure on the surface that bounds the volume in which reproduction is desired. The approach has also been studied extensively in the context of the active control of sound and vibration. 
These methods using multiple loudspeakers have been theoretically developed based on the assumption that the control area is surrounded by either continuously distributed sources, or at least uniformly distributed discrete sources in sufficient number to avoid the spatial aliasing determined by the high frequency limit to be controlled. Aside from the difficulties of positioning loudspeakers exactly, the ideal assumption of a uniform arrangement of multiple loudspeakers is clearly not met by the standard multi-channel formats such as 5.1 , 7.1, 10.2 and 22.2 channel layouts proposed by international telecommunication union [9] although there have been several research studies that consider non-uniform standard layouts $[10,11,12,13]$.

The objective of the research in this paper is to develop a control algorithm reproducing the target sound field in order to preserve the spatial perception of the virtual sources as a matter of the highest priority, regardless of loudspeaker arrangements. Dissimilar to other methods, even though the approach originates from the same assumption of continuous and discrete regular sources, the pressure matching technique using the least squares method provides a numerical approach to the solution of the "inverse problem" of determining the optimal source strengths and is not restricted to particular geometrical arrangements of sources or field points to be controlled. However, in non-uniform loudspeaker layouts, the pressure matching approach often yields results that require excessive source strength outputs. This in turn is detrimental to the perception of the location of the virtual source from which the field to be reproduced propagates. This is particularly the case when the sound field generated by the virtual source propagates from a direction from which there is either no, or a low density of loudspeakers able to render the reproduction. In order to overcome this limitation of the conventional pressure matching method, a modified method with the varying regularisation parameter dependent on the direction of the field to be reproduced has been 
proposed. This is based on the pressure matching technique and has been applied in particular to the case of a 5 channel surround system [10]. However, it is found that this approach sacrifices the performance of the system when the direction of arrival of the field to be reproduced coincides with a sufficiently high density of sources to enable good reproduction without increased regularisation. This approach also requires the magnitude of the regularization parameter to be dependent on each source arrangement, and this in turn sacrifices the system performance.

An alternative version of the least squares method for sound field reconstruction that overcomes the difficulties mentioned above has been proposed in this paper. The proposed method, the velocity matching technique, devotes the control effort to matching the particle velocities at the discrete control points on the bounding surface of the control region of the reproduced sound field with those of the target sound field. The proposed algorithm has been compared with the conventional pressure matching technique when the other conditions of control parameters applied in both methods are also comparable. Such control parameters include the regularization factor and the number and location of control points. Numerical simulations have been used to predict the performance of the new technique, which are also verified with experimental measurements. In addition, both pressure and velocity matching algorithms have been implemented in order to conduct subjective tests and assess the relative performance. These results confirm the superior capabilities of the velocity matching technique. The theory presented in this paper is based on that previously reported by the authors in a conference paper [14]. In this work, the original evaluation undertaken in the two-dimensional case [14] is extended to three dimensions with the verification of simulations, measurements and subjective tests with different designated control regions.

Section 2 defines the acoustical problem and introduces the theories of the pressure 
and velocity matching methods for the sound field reproduction. Simulation results including fields of pressure and time-averaged intensity, panning functions, and source input filters are analyzed in section 3 . Section 4 verifies the simulation results with the experimental data. Subjective test results are also presented in section 5. Based on the results presented, discussions and conclusions follow.

\section{THEORY}

The objective of sound field reproduction is to create a sound field with secondary sources (loudspeakers) that is as similar as possible to the original sound field observed at the locations of a number of control points. The input signals driving the multiple loudspeakers are obtained in order to reproduce the physical values matching the pre-observed information at the control points. Fig. 1 describes the schematic of sound reproduction system with multiple loudspeakers distributed non-uniformly.

The problem can be initially formulated based on the ideal assumption that a continuous distribution of secondary sources is available on the boundary $\partial \Lambda$ of the reproduction region, and a continuous distribution of control points (i.e. the control surface) is located on the boundary $\partial V$ of the control region. The loudspeakers are assumed to radiate sound as omnidirectional point sources. Under these assumptions, the reproduced sound field on $\partial V$ is given by the following integral formula, usually referred to as single layer potential [15, 16]:

$$
\hat{p}(\mathbf{x})=\mathrm{j} \omega \rho \int_{\mathbf{y} \in \partial \Lambda} G(\mathbf{x}, \mathbf{y}) q(\mathbf{y}) \mathrm{d} S(\mathbf{y}), \quad \mathbf{x} \in \partial V
$$

where $G(\mathbf{x}, \mathbf{y})$ is the free field Green function of the Helmholtz equation, given by 


$$
G(\mathbf{x}, \mathbf{y})=\frac{\mathrm{e}^{-\mathrm{j} k|\mathbf{y}-\mathbf{x}|}}{4 \pi|\mathbf{y}-\mathbf{x}|}
$$

and $q(\mathbf{y})$ is a function defined over $\partial \Lambda$ that determines the strengths of the continuous distribution of secondary sources. Note that all the variables are assumed to have a time dependence of $\mathrm{e}^{\mathrm{j} \omega t}$.

The particle velocity of the reproduced field is given by applying the Euler's relation [17] to Eq. (1), thus obtaining

$$
\hat{\mathbf{v}}(\mathbf{x})=-\frac{1}{\mathrm{j} \omega \rho} \nabla \hat{p}(\mathbf{x})=\mathrm{j} k \int_{\mathbf{y} \in \partial \Lambda}\left(1+\frac{1}{\mathrm{j} k|\mathbf{x}-\mathbf{y}|}\right) G(\mathbf{x}, \mathbf{y}) \mathbf{a}(\mathbf{x}, \mathbf{y}) q(\mathbf{y}) \mathrm{d} S(\mathbf{y}), \quad \mathbf{x} \in \partial V
$$

where $\mathbf{a}(\mathbf{x}, \mathbf{y})=(\mathbf{x}-\mathbf{y}) /|\mathbf{x}-\mathbf{y}|$. Clearly the particle velocity defines a vector field. The socalled normal velocity, that is a scalar quantity representing the component of the particle velocity along the direction $\mathbf{u}_{r}(\mathbf{x})$ normal to $\partial V$, is given by

$$
\hat{v}_{r}(\mathbf{x})=\mathrm{j} k \int_{\mathbf{y} \in \partial \Lambda}\left(1+\frac{1}{\mathrm{j} k|\mathbf{x}-\mathbf{y}|}\right) G(\mathbf{x}, \mathbf{y})\left[\mathbf{a}(\mathbf{x}, \mathbf{y}) \cdot \mathbf{u}_{r}(\mathbf{x})\right] q(\mathbf{y}) \mathrm{d} S(\mathbf{y}), \quad \mathbf{x} \in \partial V
$$

If either the pressure $p(\mathbf{x})$ or the normal velocity $v_{r}(\mathbf{x})$ of the target field is given, an expression for the source strength function $q(\mathbf{y})$ required to reproduce this field can be obtained by calculating a (regularized) solution of Eq. (1) and (4), which are two integral equations of the first kind. These equations define ill-posed problems, with Dirichlet or Neumann non-homogeneous boundary conditions, depending on whether the target pressure or normal velocity is given, respectively. Their solution may not exist, be non-unique, or be unstable, as explained for example in references $[16,18]$ for the sound reproduction problem or in reference [15] for the general case. This problem allows for the analytic calculation of the solution only in a limited number of cases, for which the shapes of $\Lambda$ and $V$ are such that the kernel of the integral operators can be expressed as a weighted series of orthogonal 
functions, thus allowing for an analytical inversion of the integral operator. In spite of its mathematical advantage, this analytical approach is not suitable for the majority of practical cases. The problem is therefore reformulated in a discrete setting, thus accounting for the limited number of secondary sources.

It is now assumed that $N$ secondary sources and $M$ control points are arranged on $\partial \Lambda$ and on $\partial V$, respectively. Their positions are identified by the vectors $\mathbf{y}_{n}$ and $\mathbf{x}_{m}$, respectively. The reproduced pressure and particle velocity at $\mathbf{x}_{m}, \hat{p}\left(\mathbf{x}_{m}\right)$ and $\hat{\mathbf{v}}\left(\mathbf{x}_{m}\right)$, can be represented by the source strength (or volume velocity) $q\left(\mathbf{y}_{n}\right)$ of the source located at $\mathbf{y}_{n}$, and the transfer functions regarding pressure, $Z\left(\mathbf{x}_{m} \mid \mathbf{y}_{n}\right)$, and velocity, $H\left(\mathbf{x}_{m} \mid \mathbf{y}_{n}\right)$, as shown below.

$$
\begin{aligned}
& \hat{p}\left(\mathbf{x}_{m}\right)=\sum_{n=1}^{N} Z\left(\mathbf{x}_{m} \mid \mathbf{y}_{n}\right) q\left(\mathbf{y}_{n}\right) \\
& \hat{\mathbf{v}}\left(\mathbf{x}_{m}\right)=\sum_{n=1}^{N} H\left(\mathbf{x}_{m} \mid \mathbf{y}_{n}\right) \mathbf{a}_{m, n} q\left(\mathbf{y}_{n}\right)
\end{aligned}
$$

where $\mathbf{a}_{m, n}=\left(\mathbf{x}_{m}-\mathbf{y}_{n}\right) /\left|\mathbf{x}_{m}-\mathbf{y}_{n}\right|$. The component of $\hat{\mathbf{v}}\left(\mathbf{x}_{m}\right)$ that is normal to the boundary of the control region, $\hat{v}_{r}\left(\mathbf{x}_{m}\right)$, is defined as

$$
\hat{v}_{r}\left(\mathbf{x}_{m}\right)=\sum_{n=1}^{N} H_{r}\left(\mathbf{x}_{m} \mid \mathbf{y}_{n}\right) q\left(\mathbf{y}_{n}\right),
$$

where $H_{r}\left(\mathbf{x}_{m} \mid \mathbf{y}_{n}\right)=H\left(\mathbf{x}_{m} \mid \mathbf{y}_{n}\right)\left(\mathbf{a}_{m, n} \cdot \mathbf{u}_{r}\left(\mathbf{x}_{m}\right)\right)$ and $\mathbf{u}_{r}\left(\mathbf{x}_{m}\right)$ is the unit radial inward vector normal to the surface of control region $V$ and directed towards its interior, and $\cdot$ is the inner product operator. In this work, the control region $V$ has been designed in the shape of a circle or a sphere determined by the $2 \mathrm{D}$ or $3 \mathrm{D}$ loudspeaker layout with a radius $r$ which changes as a function of frequency which helps to get the physically meaningful solution [19]. 
Considering therefore a polar or spherical coordinate system with origin in the centre of $V$, the normal velocity defined above is the opposite of the radial component of the particle velocity.

As illustrated in Fig. 1, the source strengths of all the $N$ multiple loudspeakers can be represented using a vector, $\mathbf{q}^{\mathrm{T}}=\left[\begin{array}{llll}q\left(\mathbf{y}_{1}\right) & q\left(\mathbf{y}_{2}\right) & \cdots & q\left(\mathbf{y}_{N}\right)\end{array}\right]$. The reproduced pressure and radial velocity at all the $M$ control points can be written in the form of vectors, $\hat{\mathbf{p}}^{\mathrm{T}}=\left[\begin{array}{llll}p\left(\mathbf{x}_{1}\right) & p\left(\mathbf{x}_{2}\right) & \cdots & p\left(\mathbf{x}_{M}\right)\end{array}\right]$ and $\hat{\mathbf{v}}_{r}{ }^{\mathrm{T}}=\left[\begin{array}{llll}\hat{v}_{r}\left(\mathbf{x}_{1}\right) & \hat{v}_{r}\left(\mathbf{x}_{2}\right) & \cdots & \hat{v}_{r}\left(\mathbf{x}_{m}\right)\end{array}\right]$. With the vector notations, $\hat{\mathbf{p}}$ and $\hat{\mathbf{v}}_{r}$ can be represented as below,

$$
\begin{gathered}
\hat{\mathbf{p}}=\mathbf{Z q} \\
\hat{\mathbf{v}}_{r}=\mathbf{H}_{r} \mathbf{q} \\
\text { where } \mathbf{Z}=\left[\begin{array}{ccc}
Z\left(\mathbf{x}_{1} \mid \mathbf{y}_{1}\right) & \cdots & Z\left(\mathbf{x}_{1} \mid \mathbf{y}_{N}\right) \\
\vdots & \ddots & \vdots \\
Z\left(\mathbf{x}_{M} \mid \mathbf{y}_{1}\right) & \cdots & Z\left(\mathbf{x}_{M} \mid \mathbf{y}_{N}\right)
\end{array}\right] \text { and } \mathbf{H}_{r}=\left[\begin{array}{ccc}
H_{r}\left(\mathbf{x}_{1} \mid \mathbf{y}_{1}\right) & \cdots & H_{r}\left(\mathbf{x}_{1} \mid \mathbf{y}_{N}\right) \\
\vdots & \ddots & \vdots \\
H_{r}\left(\mathbf{x}_{M} \mid \mathbf{y}_{1}\right) & \cdots & H_{r}\left(\mathbf{x}_{M} \mid \mathbf{y}_{N}\right)
\end{array}\right] \text {. }
\end{gathered}
$$

\section{A. Conventional pressure matching method}

The objective of the pressure matching method is to create a sound field with the secondary sources that is as similar as possible to the target sound field observed at the locations of control points. The input signals driving the multiple loudspeakers are required in order to reproduce the physical values matching the pre-observed pressure at the control points [8].

As the vector $\mathbf{p}$ represents all the pressure information of the target sound field at the control points, it is possible to control the loudspeaker signal inputs $\mathbf{q}$ in order to make the pressure vector $\hat{\mathbf{p}}$ of the reproduced sound field at the same location of control points as 
close as possible to $\mathbf{p}$. In other words, it is required to choose a set of loudspeaker signals $\mathbf{q}$ that minimize the distance, between the two pressure vectors, $\hat{\mathbf{p}}$ and $\mathbf{p}$ in the least squares sense. The distance is defined as

$$
d(\mathbf{p}, \hat{\mathbf{p}})=\sqrt{\sum_{m=1}^{M}\left|p\left(\mathbf{x}_{m}\right)-\hat{p}\left(\mathbf{x}_{m}\right)\right|^{2}}=\|\mathbf{p}-\mathbf{Z q}\| .
$$

The optimal source strength vector $\mathbf{q}_{0}$ that minimizes the distance $d(\mathbf{p}, \hat{\mathbf{p}})$ is given by

$$
\mathbf{q}_{0}=\left(\mathbf{Z}^{\mathrm{H}} \mathbf{Z}\right)^{-1} \mathbf{Z}^{\mathrm{H}} \mathbf{p}
$$

where the symbol $[\cdot]^{\mathrm{H}}$ and $[\cdot]^{-1}$ denote the Hermitian transpose and inverse of a matrix, respectively.

A stable solution avoiding the problem caused by the inversion of an ill-conditioned matrix can be obtained by using the technique of Tikhonov regularization [20]. The optimal solution $\mathbf{q}_{p}$ to the regularised inversion problem is given by

$$
\mathbf{q}_{p}=\left(\mathbf{Z}^{\mathrm{H}} \mathbf{Z}+\beta_{p} \mathbf{I}\right)^{-1} \mathbf{Z}^{\mathrm{H}} \mathbf{p},
$$

where $\beta_{p}$ is the regularization parameter and $\mathbf{I}$ is the $N \times N$ identity matrix.

The regularization parameter $\beta_{p}$ in Eq. (10) can make the pressure matching method applicable to sound reproduction systems having a non-uniform loudspeaker array by changing $\beta_{p}$ as a function of loudspeaker arrangement or location of virtual source [10] by applying weighting factor to the regularization parameter close to zero when the virtual source is close to the two dimensionally distributed loudspeakers, otherwise it was close to one. However, it has to involve adequate change of the regularization factor required to be tuned with respect to the arrangement of the loudspeakers used in every sound system. In 
addition, an unnecessarily imposed regularization parameter $\beta_{p}$ may sacrifice the performance of the sound reproduction.

\section{B. Proposed velocity matching method}

It is clear that if one is concerned to reproduce a sound field with the objective of ensuring a realistic perception of the location of the source of that sound field, then it makes sense to ensure the reproduction of the direction of travel of the sound. A convenient measure of the magnitude and direction of energy flow in an arbitrary sound field is provided by the timeaveraged acoustic intensity. The intensity will therefore be used here as another useful physical measure of the accuracy to which a sound field can be reproduced [5, 21, 22]. The time averaged intensity, which is of course a vector quantity having magnitude and direction, can be defined for steady state fields as [17],

$$
\mathbf{I}\left(\mathbf{x}_{m}\right)=\frac{1}{2} \operatorname{Re}\left(p\left(\mathbf{x}_{m}\right) \mathbf{v}\left(\mathbf{x}_{m}\right)^{*}\right)
$$

where $\mathbf{v}\left(\mathbf{x}_{m}\right)$ is the velocity vector of the target sound field at $\mathbf{x}_{m}, \operatorname{Re}(\cdot)$ stands for the real part and ${ }^{*}$ for complex conjugate. Eq. (11) shows that the direction of the intensity can be controlled by controlling the velocity vector only. However, unlike the pressure, it is not straightforward to apply the least squares method to velocity (which is a vector).

The velocity is a vector quantity which has two components such as the amplitude and the direction. It means that the physical parameters considered to be controlled should be more than one depending on the loudspeaker layouts whether they are arranged in 2D or 3D space. One simple way to control this vector quantity regardless of the loudspeaker layouts is to control the amplitude of the radial components which is normal to the surface of the 
control region in shape of circle for $2 \mathrm{D}$ or sphere for 3D loudspeaker configuration. The cost function for this control method is in the same formulation simply replacing the pressure with the amplitude of the radial velocity components. In addition, sound field within a control region can be exactly reproduced when you can control inputs to generate the velocity components normal to the control surface. As the control region is a circle or a sphere, the normal velocity components are the radials, $v_{r}\left(\mathbf{x}_{m}\right)=\mathbf{v}\left(\mathbf{x}_{m}\right) \cdot \mathbf{u}_{r}\left(\mathbf{x}_{m}\right)$.

When $\mathbf{v}_{r}$ is the vector of target radial velocity at all control points, the distance between $\mathbf{v}_{r}$ and $\hat{\mathbf{v}}_{r}$ can be written as the same form of Eq. (8). The optimal solution $\mathbf{q}_{v}$ to the regularised inversion problem is also given as the same form of Eq. (10) by

$$
\mathbf{q}_{v}=\left(\mathbf{H}_{r}{ }^{\mathrm{H}} \mathbf{H}_{r}+\beta_{v} \mathbf{I}\right)^{-1} \mathbf{H}_{r}{ }^{\mathrm{H}} \mathbf{p},
$$

where $\beta_{v}$ is the regularization parameter of the proposed method.

\section{SOUND FIELD SIMULATIONS}

Throughout the simulations described in this section, the pressure transfer function from each point source at $\mathbf{y}$ to any field point $\mathbf{x}$, for a given wave number $k$, is represented by the freefield Green function multiplied by j $\omega \rho$ referred by Eq. (1) and is given by

$$
Z(\mathbf{x} \mid \mathbf{y})=\frac{\mathrm{j} \omega \rho}{4 \pi} \frac{\mathrm{e}^{-\mathrm{j} k d}}{d}
$$

where $d=|\mathbf{x}-\mathbf{y}|$. The corresponding velocity transfer function is given by

$$
H(\mathbf{x} \mid \mathbf{y})=\frac{\mathrm{j} k \mathrm{e}^{-\mathrm{j} k d}}{4 \pi d}\left(1+\frac{1}{\mathrm{j} k d}\right)
$$

Note that the same transfer functions of pressure and velocity have been used regardless of 
the loudspeaker arrangements.

As described in Fig. 2, numerical simulations for the 3D loudspeaker arrangement have been carried out using 22 channels based on the cylindrical layout of 22.2 channel system proposed by NHK (Nippon Hōsō Kyōka: Japan Broadcasting Corporation) [23] without the two low-frequency effect channels, and 5 channels which are a subset of the 22 channels and are used here as an application of a 2D loudspeaker arrangement. The actual source locations of each multichannel system are listed in Table I.

Fig. 3 represents the three locations of the virtual spherical sound sources at the 2 metre distance from the origin in 3D (22 channel) and 2D (5 channel) loudspeaker configurations. The detailed coordinates of the three spherical virtual sources are also shown in Table II. This also depicts the area of the simulated plane and the location of the control points. In this section, 144 points equidistantly spaced on the sphere have been used as control points for 3D loudspeaker arrangements and 32 points on the circle for 2D loudspeaker layouts. The radii of the control sphere, $r_{3 D}$ and circle, $r_{2 D}$ have been set as the frequency dependant radius defined as,

$$
r_{3 D}=c(\sqrt{N}-1) / \omega, \quad r_{2 D}=c(N-1) / 2 \omega
$$

where $c$ is the speed of sound. This reduces the condition number of the matrix to be inverted, thus improving the robustness of the solution [24, 25].

Both pressure and velocity matching methods uses the normalized Tikhonov regularization factors calculated from [26, 27],

$$
\beta=\beta_{0} \sigma_{1}^{2}
$$

where $\sigma_{1}$ is the largest singular value of the transfer function matrix $\mathbf{Z}$, and the parameter $\beta_{0}$ is a real positive constant number. We used $\beta_{0}=10^{-4}$ in this paper for comparing the 
conventional and proposed algorithms. It makes the effort to choose the regularization factor same by using the normalized regularization factor which has been used in the conventional algorithm.

The temporal and spatial sampling rates for all the simulation are $48 \mathrm{kHz}$ and 0.001 metres, respectively.

\section{A. Reproduction of pressure}

Fig. 4 shows the target pressure fields caused by the three spherical virtual sources at positions $\mathrm{A}, \mathrm{B}$ and $\mathrm{C}$ in Fig. 3 (a) and also represents the reproduced pressure fields controlled by pressure and velocity control methods with the 22 channel 3D loudspeaker configuration. The excitation signal is a single frequency at $1000 \mathrm{~Hz}$. The reproduced pressure fields controlled by both methods are similar to each other when the target spherical

sources located at A in Fig. 3 (a). In this case there are comparatively many loudspeakers arranged with small gaps between adjacent loudspeakers that are able to easily reproduce the target field. However, when the target source is located at positions B and C, in which directions there are fewer loudspeakers arranged with large gaps between them, the pressure fields controlled by the pressure matching method become unstable due to excessively large source outputs. However, the velocity matching method results in similar pressure amplitude levels regardless of the source location. Even though the stabilized solutions can be obtained by increasing the regularization parameter in the process of pressure control method, the results cannot be identical to the velocity controlled ones. Because control of the regularization parameter, a type of numerical damping for the stable solutions, has no conceptual similarity with the physically motivated velocity matching method. On the other hand, the proposed velocity control method provides the controlled results with minimum 
care for the regularization parameter.

The mismatch has been defined as the Pressure Error (PE) between pressures in one pressure field, $p$ (of the target field) and another field, $\hat{p}$ (of the reproduced field) as given by

$$
\mathrm{PE}=\frac{|p-\hat{p}|^{2}}{|p|^{2}} \times 100(\%) .
$$

It is noted that the PE is the measure of the pressure mismatch considering both amplitude and phase. Fig. 5 represents the mismatch of the reproduced pressure fields relative to the target fields based on the PE calculations on the simulated plane. When the virtual source is in a direction where many loudspeakers are located, the area with smaller PE is wider than the others. The virtual sources positioned at B and C, where only a few loudspeakers are arranged, results in the narrower regions of smaller PE compared with position A. With application of the proposed velocity matching method, a wider area with less pressure error has been achieved.

As for the 2D configuration, Fig. 6 shows the target pressure fields due to the virtual sources in Fig. 3 (b) including the simulated reproduced pressure fields of both control algorithms with 5 channels. With the increased gap between the adjacent loudspeakers, similar tendencies to those shown in Fig. 4 have been observed with larger source output signals causing severe instability. Fig. 7 represents the mismatch of the reproduced pressure fields relative to the target fields in the case of the five channel 2D source arrangement. The same tendencies have been observed as those exhibited by the $3 \mathrm{D}$ configuration of loudspeakers.

As a result, except for a slight increase of area of small PE, there is no noticeable improvement in terms of PE by applying the proposed velocity matching method although 
significant level of stabilization of the source output signals has been achieved.

\section{B. Reproduction of intensity direction}

Following the discussion of intensity in section 2 as a potentially useful measure of the quality of reproduction of a sound field, the concept of normalized unit intensity vector obtained by $\mathbf{u}^{\mathrm{I}}=\mathbf{I} /|\mathbf{I}|$ has been adopted to describe the direction of the energy flow at certain location on the simulated planes. It can be also a clear measure for the phase mismatch at the positions of the reproduced field controlled by the individual input signals. The Intensity Direction Error (IDE) can be defined by the mismatch between the normalized unit intensity vectors of the target and reproduced wave fields, which relates to the difference in angles between those two vectors. The IDE of a unit intensity vector, $\hat{\mathbf{u}}^{\mathrm{I}}$ (of the reproduced fields) to another one, $\mathbf{u}^{\mathrm{I}}$ (of the target field) can be obtained by the calculation below,

$$
\mathrm{IDE}=\operatorname{acos}\left(\mathbf{u}^{\mathrm{I}} \cdot \hat{\mathbf{u}}^{\mathrm{I}}\right) / \pi \times 100(\%)
$$

where $\cdot$ denotes the inner product of two vectors. Due to the consistency with the PE, the IDE has been represented as percentage value. $0 \%$ of the IDE means the same direction $\left(0^{\circ}\right)$ and $100 \%$ of the IDE means the opposite direction $\left(180^{\circ}\right)$ between the reproduced and the reference intensity vectors.

Based on the calculation using IDE, Fig. 8 shows the mismatch of the intensity directions of the reproduced fields compared with the target field in the case of the 22 channel loudspeaker system. By changing the control algorithm from the conventional pressure matching method to the proposed velocity matching method, the IDE has been decreased, especially when the target source is at positions $\mathrm{B}$ and $\mathrm{C}$, where fewer loudspeakers are located. In Fig. 9, similar trends have been observed as for the 22 channel 
system case.

As for the inter-channel comparison between 22 and 5 channel sound systems using the two IDE field plots of Fig. 8 and 9, it is obvious that the introduction of the larger gap between adjacent loudspeakers causes a larger variation of IDE with respect to the virtual source position due to the excessive source output problem. However, the proposed velocity matching method provides stability of the IDE regardless of the virtual source position. In addition, Fig. 10. Represents the averaged IDE over the simulated plane with respect to the change of frequencies over the two different loudspeaker layouts controlled by the two different methods when there are three different target sources. Fig. 10 (a) and (b) shows the overall reduction of IDE in case of 22 channel loudspeaker layout. With 5 channel loudspeaker layout, a noticeable improvement below $3000 \mathrm{~Hz}$ has been observed when the target source is located in $\mathrm{C}$ position along with the overall reduction of IDE.

\section{Panning function}

The source strengths associated with all the loudspeakers with respect to the angle of virtual source location over the full range of incidence angles, which we refer to here as the panning functions, have been computed at the same frequency of $1000 \mathrm{~Hz}$. Those are obtained for both the pressure and velocity matching techniques when the elevation angle $(\theta)$ of virtual source is fixed at $90^{\circ}$ corresponding to the horizontal plane. The panning functions of the 22 and 5 channel systems are shown in Fig. 11 and overlaid with the $\ell^{2}$ norm of all of the sources (which gives an indication of the total energy consumed in reproduction). The panning functions associated with the velocity matching method in Fig. 11 (c) and (d) shows a more stable distribution of the magnitudes of the source strengths than those obtained by the pressure matching method in Fig. 11 (a) and (b), regardless of the angle of virtual sources 
for both channel configurations. Consequently, the $\ell^{2}$ norm of the source strengths controlled by the velocity matching method shows much less variation than that of the pressure matching method. The velocity control method uses all the possible loudspeakers close to the location of the virtual source when the virtual source is located in the areas that are densely populated by loudspeakers. It also uses only a few loudspeakers well aligned with the direction of the incident sound to be reproduced when the virtual source is located in the area that are sparsely populated by loudspeakers. Fig. 11 (e) and (f) represents the $\ell^{2}$ norm of all source output signals in polar coordinates together with the actual location of the loudspeakers. In the case of the pressure matching method, the changes of the required energy with respect to the location of the loudspeakers have been clearly observed whilst the velocity matching method resolved the severe dependency of output energy on the loudspeaker locations and maintains this at an almost constant value. Based on the panning functions of the pressure control method, it is obvious that the optimal source strengths show excessive output power whenever the virtual source is located in the areas that are sparsely populated by loudspeakers. If the virtual source is in a densely populated region or at an angle corresponding to a secondary source location, the optimal solutions for the source strengths associated with the pressure control method do not suffer from the problem of excessive power. However, the panning functions associated with the velocity control method produce stable source energies due to the reasonable source strength distributions throughout the entire range of incidence angles. Based on these observations of the panning functions, it can be said that the advantage of the velocity control method is the good adaptability to any loudspeaker layouts without further control effort. Fig. 12 represents the corresponding phase changes of inputs with respect to the direction of target source in case of 22 and 5 channel systems controlled by both pressure and velocity matching methods. 


\section{Input filters}

Fig. 13 shows the frequency dependency of the two techniques by computing the filters which are neecsseary to process the input signal associated with the virtaul source located in position $\mathrm{C}$ in Fig. 3. Results are shown for the cases where the loudspeakers are sparsely located in both the 22 and 5 channel systems. Note that these filters have been equalized by a factor, $j \omega \rho_{0}$. The total required energies, that is the $\ell^{2}$ norms of both control method, have been also represented with black dotted lines. The filters generated by the pressure control method suffer from the excessive power problem within the crucial frequency range from around 300 to $2000 \mathrm{~Hz}$. However, the $\ell^{2}$ norms of the filters generated by the velocity control method are flatter than the conventional method and this is likely to result in sound reproduction without undue coloration of frequency components.

\section{SOUND FIELD EXPERIMENTS}

The simulated results presented in the previous section are validated by measuring the sound field generated through the real cylindrical NHK 22.2 channel system (without 0.2 subwoofers) built in an anechoic chamber of the Institute Sound and Vibration Research (ISVR). The RME multichannel audio system composed of a HDSP MADI, ADI-648 and five ADI-8 DS has been used for audio interface of 22 loudspeakers (KEF HTS3001) and 34 microphones (Brüel\&Kjær 1/2 inch Type 4189). Fig. 14 describes the 22 loudspeakers allocated regarding the positions in Table I and Fig. 3 (a). For 5 channel system, all the other loudspeakers were turned off except the 5 loudspeakers listed in Table I. The level of loudspeakers was calibrated to produce the same sound pressure level at the centre of the 
loudspeaker system within an error of $\pm 1 \mathrm{~dB}$. The linear microphone array with uniformly spaced 36 microphones (the $0.051 \mathrm{~m}$ gap between adjacent microphones) is shown in Fig. 15. All microphones were calibrated relative to a reference microphone.

The input signals for all the loudspeakers were white noise passed through the input filters generated by both control methods. The reproduced sound fields by both pressure and velocity methods have been captured by using the linear microphone array discretely translated on the horizontal plane as shown in Fig. 3 with $0.05 \mathrm{~m}$ interval. The measured plane covers the simulated plane by 1188 points ( 36 microphones $\times 33$ translation positions). In correspondence with the simulation results, the sampling rate adopted is $48 \mathrm{kHz}$ for signal inputs to all of the loudspeakers.

Fig. 16 represents the band-passed measured pressure fields reproduced by using the 22 channel system controlled by the two control algorithms, pressure and velocity matching methods. Their mismatches to the simulated fields controlled by both methods shown in Fig. 4 have been represented in Fig. 17. The measured pressure fields in case of 5 channel system are shown in Fig. 18 and they have been also compared with the simulated fields controlled by both methods in Fig. 6 with the resulting mismatch plot shown in Fig. 19. With the observation of error fields between simulated and measured pressure fields, the measurement results of the velocity control method show better matching with the simulated results than those of the pressure control method. Consequently, it is clear that the velocity matching algorithm for sound field reproduction is more robust than the pressure control method to input errors such as those caused by inaccurate loudspeaker arrangement, noise on the input signals and radiation directivity variation of the loudspeakers. 


\section{PERCEPTUAL LOCALIZATION TESTS}

A simple subjective test with 15 normal hearing subjects aged from 25 to 35 has been designed to validate the theory, simulations and experiments described above and to evaluate the improvement of perceptual localization accuracy produced by using the proposed velocity matching method. A Polhemus FASTRAK® was used as the device for collecting the positions of the subject's sound localization due to its capability of real-time tracking in 6 degrees of freedom. The reference transmitter was fixed on the floor and the receiver mounted on a rod for the subject to hold and to point towards the perceived direction of the virtual source. Each subject was trained with a calibration session which involved pointing to 8 angles $\left((\phi, \theta)=\left(45^{\circ} \times(n-1), 90^{\circ}\right)\right.$ where $\left.n=1, \ldots, 8\right)$ in order to ensure the reliability of the pointing device prior to the actual subjective tests and minimize the error induced by the pointing method [26]. The subjects were instructed to fix their heads and bodies in order to look in the frontal direction during the period when the stimuli were presented. A white noise signal of five seconds duration was used as a source signal and this was processed to create the loudspeaker input signals. These signals were intended to generate reproduced sound fields associated with the three virtual source positions listed in Table II for both pressure and velocity matching methods. The rendered sound fields corresponding to the range of locations of the virtual sources were presented to the subjects in randomized order of the virtual source locations and control methods. In other words, 12 cases composed of two different loudspeaker layouts (5 and 22 channels), three different virtual source positions (A, B, C) and two control methods (pressure and velocity matching methods) have been randomly presented to the test subjects. In each case, the test period was ten seconds including five second signal duration and the same length of pause. Note that the test results from 15 have been considered for the representation of the localization performance and the 
loudspeakers were hidden from the sight of test subjects by blind-folding the subjects.

Fig. 20 shows $5 \sim 95$ percentile ranks which are described by lines in the cases of 22 (a, b, c) and 5 (d, e, f) channel systems. Also indicated with circle is the 50 percentile rank or median value. The blue colored data represents the subjective test results when the pressure matching method is applied while the red colored data shows those of the velocity matching method. The percentile rank has been used for representing the results due to the relatively small number of subjects and resulting test data. The percentile rank does not consider any predefined distribution of the sample data set. It is regarded as a more representative expression of the proposed subjective test results than using mean and standard deviation, which assume a Gaussian distribution of the data set [29].

The subjective test results produced by using both pressure and velocity matching methods with the 22 channel system are shown in Fig. 20 (a, b, c). These are very similar when the virtual source is located at position A that is densely populated by loudspeakers. When the virtual sources are located at positions B and C, where loudspeakers are relatively sparse, the subjects within the reproduced sound field controlled by the velocity matching method always localize the virtual sources closer than with the pressure matching method. In case of source position $\mathrm{B}$, the subjective test results obtained by the velocity matching method using the 5 channel system also shows closer localization performance in terms of median value, although with slightly larger variations indicated by the angular range of the curve than the pressure matching methods. At the source position $\mathrm{C}$, where there are few loudspeakers, the subjects in the sound field reproduced by velocity control method localize the source position with less confusion than with the pressure matching method. These subjective test results can be regarded as an additional support for the motivation of utilizing the intensity vector for improving localization performance of human hearing. 


\section{DISCUSSION}

When loudspeakers are distributed sufficiently uniformly, the exact reproduction of the pressure field on the bounding surface of the control volume clearly provides an effective method of reproduction. However it is also clear that this technique has limitations due to spatial aliasing when the distribution of loudspeakers is relatively sparse and/or irregular. Different from the pressure matching method, the proposed velocity matching method have no limit for the aliasing frequency because it controls the energy flow instead of matching the pressures on the control surface.

The velocity matching method can be regarded as an alternative algorithm inbetween the pressure matching method and simple amplitude panning method. When there are enough loudspeakers in the direction of virtual source, the velocity control method shows similar performance to the pressure control method. However, the performance of the velocity control method shows a performance that has some of the attributes of the simple amplitude panning method when there are few loudspeakers in the direction of the virtual source location.

The velocity control method also achieves good control of the sound intensity flow in the reproduced field. When the number of loudspeakers is sufficient, there is no noticeable difference between the pressure and velocity control techniques. However, it appears that there is some improvement of human sound localization performance when the intensity is more effectively controlled in the case of sparse and irregular loudspeaker distributions.

It is arguable that a similar effect can be produced by controlling the Tikhonov regularization parameter in the pressure matching method. This implies that the regularization 
parameter should be tuned based on the location of the virtual source considering the density of the loudspeakers allocated in the multichannel sound system. However, by controlling velocity instead of pressure at the same control points, it appears that a single normalized regularization parameter is sufficient regardless of the arrangement of the loudspeakers and the location of the virtual sources.

\section{CONCLUSIONS}

The main purpose of this paper has been to propose an alternative sound reproduction algorithm which can achieve a certain level of localization of virtual sound sources based on the better match of intensity directions even when the loudspeaker arrangements used are relatively sparse and non-uniform.

This challenge has led to the development of a new sound field reproduction technique based on the velocity matching method. This is implemented by matching the normal particle velocity components in the target and reproduced fields on the surface of the control volume. It is also important that the size of the control volume is made frequency dependent. To verify the performance of the method developed, both the conventional pressure matching method and the proposed velocity matching method have been implemented in three and two dimensional loudspeaker arrangements respectively consisting of 22 and 5 non-uniformly distributed loudspeakers. The advantages of the velocity matching method have been demonstrated with numerical simulations, objective measurements and subjective tests. Based on the results presented, the method proposed here confers an alternative robust solution for reproducing a sound field irrespective of the location of the virtual source when there are limited numbers of loudspeakers non-uniformly spaced. The 
results demonstrated show performance improvements when compared with the conventional pressure matching method, in terms of the reconstructed sound pressure, intensity direction error, panning function, and source input frequency response function. These improvements are evident in the simulations and corresponding experiments presented. It is also partly supported by the subjective test results.

\section{ACKNOWLEDGEMENTS}

This work was partially supported by the IT R\&D program (2008-F-011, Development of Next Generation DTV Core Technology) of KEIT, KCC and MKE, Korea, by the Royal Academy of Engineering and by the Engineering and Physical Sciences Research Council, United Kingdom of Great Britain and Northern Ireland (10216/85).

\section{REFERENCES}

[1] H. Moller, "Fundamentals of Binaural Technology," Applied Acoustics, 36, 171-218 (1992).

[2] T. Takeuchi, P. A. Nelson, "Optimal source distribution for binaural synthesis over loudspeakers," Journal of the Acoustical Society of America, 112, 2786-2797 (2002).

[3] A. J. Berkhout, D. de Vries, P. Vogel, "Acoustic control by wavefield synthesis," Journal of the Acoustical Society of America, 93, 2764-2778 (1993).

[4] P. Gauthier, A. Berry, "Adaptive wave field synthesis with independent radiation mode control for active sound field reproduction: Theory," Journal of the Acoustical Society of America, 119 (5), 2721-2737 (2006). 
[5] M. A. Gerzon, "Periphony : With-height sound reproduction," Journal of the Audio Engineering Society, 21 (1), 2-10 (1973).

[6] D. B. Ward, T. D. Abhayapala, "Reproduction of a Plane-Wave Sound Field Using an Array of Loudspeakers," IEEE Transaction on Speech and Audio Processing, 9, 697-707 (2001).

[7] P. A. Nelson, S. J. Elliott, Active Control of Sound, Academic, London (1992).

[8] O. Kirkeby, P. A. Nelson, "Reproduction of plane wave sound fields," Journal of the Acoustical Society of America, 94 (5), 2992-3000 (1993).

[9] ITU-R Rec. BS. 6C/253-E, “Three-dimensional multichannel stereophonic sound system for programme production, proposed Preliminary draft new recommendation itu-r bs.[3D-Sound]," Geneva, 26 October (2009).

[10] M. A. Poletti, "Robust two-dimensional surround sound reproduction for nonuniform loudspeaker layouts," Journal of the Audio Engineering Society, 55 (7/8), 598-610 (2007).

[11] P. Craven, "Continuous Surround Panning for 5-speaker Reproduction,” Proceedings of the 24th International conference of Audio Engineering Society, Banff, Canada (2003).

[12] B. Wiggins, "The Generation of Panning Laws for Irregular Speaker Arrays Using Heuristic Methods," Proceedings of the 31st International conference of Audio Engineering Society, London, United Kingdom (2007).

[13] A. Ando, "Conversion of reproduced sound field based on the coincidence of sound pressure and direction of particle," Proceedings of 20th International Congress on Acoustics, Sydney, Australia (2010). 
[14] M. Shin, F. M. Fazi, P. A. Nelson, J. Seo, "Control of velocity for sound field reproduction", Proceedings of the 52nd International conference of Audio Engineering Society, Guildford, United Kingdom (2013)

[15] D. L. Colton, R. Kress, Inverse Acoustic and Electromagnetic Scattering Theory, Springer, Berlin (1992).

[16] F. M. Fazi, Sound field reproduction. University of Southampton, Institute of Sound and Vibration Research, Doctoral Thesis (2010).

[17] E. G. Williams, Fourier acoustics: Sound radiation and near-field acoustical holography, Academic Press, London (1999).

[18] F. M. Fazi, P. A. Nelson, "Nonuniqueness of the solution of the sound field reproduction problem with boundary pressure control," Acta Acustica united with Acustica, 98 (1), 1-14. (2012).

[19] F. M. Fazi, P. A. Nelson, J. E. N. Christensen, J. Seo, "Surround system based on three-dimensional sound field reconstruction," Proceedings of the 125th Convention of the Audio Engineering Society, San Francisco, CA (2008).

[20] P. C. Hansen, Rank-Deficient and Discrete Ill-posed Problems, SIAM, Philadelphia (1998).

[21] J. Daniel, J. B. Rault and J. D. Polack, "Ambisonics Encoding of Other Audio Formats for Multiple Listening Conditions," Proceedings of the 105th Convention of the Audio Engineering Society, San Francisco, CA (1998).

[22] J. Merimaa and V. Pulkki, "Spatial Impulse Response Rendering I: Analysis and Synthesis," Journal of the Audio Engineering Society, 53 (12), 1115-1127 (2005). 
[23] K. Hamasaki, T. Nishiguchi, R. Okumura, Y. Nakayama, A. Ando, "A 22.2 Multichannel Sound System for Ultrahigh-Definition TV (UHDTV)," SMPTE Motion Imaging Journal, 117(3), 40-49 (2008).

[24] F. M. Fazi, P. A. Nelson, J. Seo, K. Kang, "Apparatus and method for sound reproduction," UK patent pending, GB application No. 0817950.9, (2008).

[25] R. A. Kennedy, P. Sadeghi, T. D. Abhayapala, H. M. Jones, "Intrinsic limits of dimensionality and richness in random multipath fields," IEEE Transaction on Speech and Audio Processing, 55 (6), 1003-1010 (2007).

[26] P. Gauthier, C. Camier, O. Gauthier, Y. Pasco, A. Berry, "Sound field reproduction of real flight recordings in cabin mock-up: Modified multichannel equalization," Proceedings of the 52nd International conference of Audio Engineering Society, Guilford, United Kingdom (2013).

[27] M. Shin, F. M. Fazi, P. A. Nelson, F. Casagrande Hirono, "Controlled sound field with a dual layer loudspeaker array," Journal of Sound and Vibration, 333 (16), 3794-3817, (2014).

[28] P. Majdak, B. Laback, M. Goupell, M. Mihocic, "The accuracy of localizing virtual sound sources: effects of pointing method and visual environment," Proceedings of the 124th Convention of the Audio Engineering Society, Amsterdam, Netherlands (2008).

[29] L. Crocker, J. Algina, Introduction to classical and modern test theory, Harcourt Brace Jovanovich College Publishers, New York (1986). 


\section{Captions for figures:}

Fig. 1. A schematic of the sound reproduction system with a non-uniform loudspeaker distribution. The volume velocity of the equivalent source at a loudspeaker location is denoted by $q\left(\mathbf{y}_{n}\right)$ whilst $\hat{p}\left(\mathbf{x}_{m}\right)$ and $\hat{\mathbf{v}}\left(\mathbf{x}_{m}\right)$ respectively denote the reproduced pressure and particle velocity vector at a control point on the boundary of the control region. The control region has radius $r$ and volume $V$ and is contained within the reproduction region $\boldsymbol{\Lambda}$.

Fig. 2. The spherical coordinate system and the source distribution of the 22 channel system

Fig. 3. Geometrical arrangements and the locations of virtual spherical sources and control points for (a) 3D and (b) 2D loudspeaker arrangements. The planes used for illustrating the variables computed in the 3D and 2D simulations are also shown.

Fig. 4. Target pressure fields $(a, b, c)$ induced by the three virtual spherical sources at the 
positions A, B, C shown in Fig. 3 in the case of the 22 channel loudspeaker system. The corresponding simulated reproduced pressure fields controlled by the pressure matching method are shown in $(\mathrm{d}, \mathrm{e}, \mathrm{f})$ and those produced by using the velocity matching method are shown in $(g, h, i)$.

Fig. 5. Differences between the target pressure fields and the reproduced fields controlled by the pressure matching method $(a, b, c)$ and the velocity matching method $(d, e, f)$ in the case of 22 channel loudspeaker system shown in Fig. 3.

Fig. 6. Target pressure fields $(a, b, c)$ induced by the three virtual spherical sources at A, B, C and corresponding simulated reproduced pressure fields controlled by the pressure matching method (d, e, f) and the velocity matching method $(g, h, i)$ in case of the 5 channel loudspeaker system shown in Fig. 3. (Some of these results were first presented in reference [14]).

Fig. 7. Differences between the reproduced and target pressure fields controlled by the pressure matching method $(a, b, c)$ and the velocity matching method $(d, e, f)$ in the case of the 5 channel loudspeaker system shown in Fig. 3. (Some of these results were first presented in reference [14]).

Fig. 8. Differences between the intensity directions of the target and the reproduced pressure fields controlled by the pressure matching method $(a, b, c)$ and the velocity matching method $(d, e, f)$ in the case of 22 channel loudspeaker system shown in Fig. 3. 
Fig. 9. Differences between the intensity directions of the target and the reproduced pressure fields controlled by the pressure matching method $(a, b, c)$ and the velocity matching method (d, e, f) in the case of the 5 channel loudspeaker system shown in Fig. 3. (Some of these results were first presented in reference [14]).

Fig. 10. Averaged IDE over the simulated plane with respect to the frequency of 22 channel (a), (b) and 5 channel (c), (d) loudspeaker systems controlled by the pressure (a), (c) and the velocity control methods (b), (d) when the target sources are located at the positions, A, B, C in Table II.

Fig. 11. Panning functions of each input in the 22 (a, c, e) and 5 (b, d, f) channel systems controlled by the pressure $(\mathrm{a}, \mathrm{b})$ and the velocity $(\mathrm{c}, \mathrm{d})$ matching methods. The $\ell^{2}$ norms of all the source inputs associated with the pressure (solid line) and velocity (dashed lines) matching methods have been compared in polar plots $(e, f)$ with the actual location of loudspeakers depicted by black circles (0).

Fig. 12. Phase change associated with each source input with respect to the direction of the target source in the $22(\mathrm{a}, \mathrm{c})$ and $5(\mathrm{~b}, \mathrm{~d})$ channel systems controlled by the pressure $(\mathrm{a}, \mathrm{b})$ and the velocity (c, d) matching methods.

Fig. 13. Optimum input filters of all the sources obtained by the pressure $(a, c)$ and the velocity (b, d) matching methods when the virtual source is located in position $\mathrm{C}$ in Fig. 3 of the $22(a, b)$ and $5(c, d)$ channel layouts. The $\ell^{2}$ norms of the source inputs are depicted with the dashed lines. 
FIG. 14. Experimental setup for cylindrical NHK 22 channel system installed in the ISVR anechoic chamber

FIG. 15. Measurement equipment arrangement using a linear microphone array composed of 36 condenser microphones.

Fig. 16. Measured sound fields reproduced by pressure (a, b, c) and velocity (d, e, f) matching methods corresponding to the simulations in Fig. 4 in the case of the 22 channel system.

Fig. 17. Differences between the measured and the simulated pressure fields in Fig. 4 controlled by the pressure matching method $(a, b, c)$ and the velocity matching method $(d, e, f)$ in the case of the 22 channel loudspeaker system.

Fig. 18. Measured sound fields reproduced by the pressure (a, b, c) and the velocity (d, e, f) matching methods corresponding to the simulations in Fig. 6 in the case of the 5 channel system. (Some of these results were first presented in reference [14]).

Fig. 19. Differences between the measured and simulated pressure fields in Fig. 6 controlled by the pressure matching method $(a, b, c)$ and the velocity matching method $(d, e, f)$ in the case of the 5 channel loudspeaker system. (Some of these results were first presented in reference [14]). 
Fig. 20. Results of the subjective localization performance in the case of the $22(\mathrm{a}, \mathrm{b}, \mathrm{c})$ and the $5(\mathrm{~d}, \mathrm{e}, \mathrm{f})$ channel systems controlled by pressure (blue) and velocity (red) matching methods when the loudspeakers are arranged at the black circles (o) and the virtual sources are located at the crosses (+) where A, B, C are located as described in Table II.

Table I. Source positions of the 22 and 5 channel systems

\begin{tabular}{|c|c|c|c|c|c|c|}
\hline \multirow{2}{*}{ Layer } & \multirow{2}{*}{$\begin{array}{l}\text { Source } \\
\text { Number }\end{array}$} & \multirow{2}{*}{$\rho[\mathrm{m}]$} & \multirow{2}{*}{$\theta\left[{ }^{\circ}\right]$} & \multirow{2}{*}{$\phi\left[^{\circ}\right]$} & \multicolumn{2}{|c|}{ System } \\
\hline & & & & & $22 \mathrm{Ch}$. & $5 \mathrm{Ch}$. \\
\hline \multirow{9}{*}{$\begin{array}{l}\text { Top } \\
\text { Layer }\end{array}$} & 1 & 0.97 & 0 & 0 & 1 & \\
\hline & 2 & 1.55 & 51.3 & 0 & 2 & \\
\hline & 3 & 1.55 & 51.3 & 45 & 3 & \\
\hline & 4 & 1.55 & 51.3 & 90 & 4 & \\
\hline & 5 & 1.55 & 51.3 & 135 & 5 & \\
\hline & 6 & 1.55 & 51.3 & 180 & 6 & \\
\hline & 7 & 1.55 & 51.3 & 225 & 7 & \\
\hline & 8 & 1.55 & 51.3 & 270 & 8 & \\
\hline & 9 & 1.55 & 51.3 & 315 & 9 & \\
\hline \multirow{10}{*}{$\begin{array}{l}\text { Middle } \\
\text { Layer }\end{array}$} & 10 & 1.21 & 90.0 & 0 & 10 & 10 \\
\hline & 11 & 1.21 & 90.0 & 22.5 & 11 & \\
\hline & 12 & 1.21 & 90.0 & 45 & 12 & 12 \\
\hline & 13 & 1.21 & 90.0 & 90 & 13 & \\
\hline & 14 & 1.21 & 90.0 & 135 & 14 & 14 \\
\hline & 15 & 1.21 & 90.0 & 180 & 15 & \\
\hline & 16 & 1.21 & 90.0 & 225 & 16 & 16 \\
\hline & 17 & 1.21 & 90.0 & 270 & 17 & \\
\hline & 18 & 1.21 & 90.0 & 315 & 18 & 18 \\
\hline & 19 & 1.21 & 90.0 & 337.5 & 19 & \\
\hline \multirow{3}{*}{$\begin{array}{l}\text { Bottom } \\
\text { Layer }\end{array}$} & 20 & 1.34 & 115.0 & 0 & 20 & \\
\hline & 21 & 1.34 & 115.0 & 45 & 21 & \\
\hline & 22 & 1.34 & 115.0 & 315 & 22 & \\
\hline
\end{tabular}


Table II. Three spherical virtual source locations

\begin{tabular}{cccccc}
\hline \multirow{2}{*}{$\begin{array}{c}\text { Virtual } \\
\text { Source }\end{array}$} & $\rho[\mathrm{m}]$ & \multicolumn{2}{c}{$22 \mathrm{Ch}}$. & \multicolumn{2}{c}{$5 \mathrm{Ch}}$. \\
\cline { 3 - 6 } & & $\theta\left[^{\circ}\right]$ & $\phi\left[^{\circ}\right]$ & $\theta\left[^{\circ}\right]$ & $\phi\left[^{\circ}\right]$ \\
\hline $\mathrm{A}$ & 2 & 90 & 15 & 90 & 15 \\
\hline $\mathrm{B}$ & 2 & 80 & 80 & 90 & 80 \\
\hline $\mathrm{C}$ & 2 & 70 & 165 & 90 & 180 \\
\hline
\end{tabular}


Click here to download high resolution image

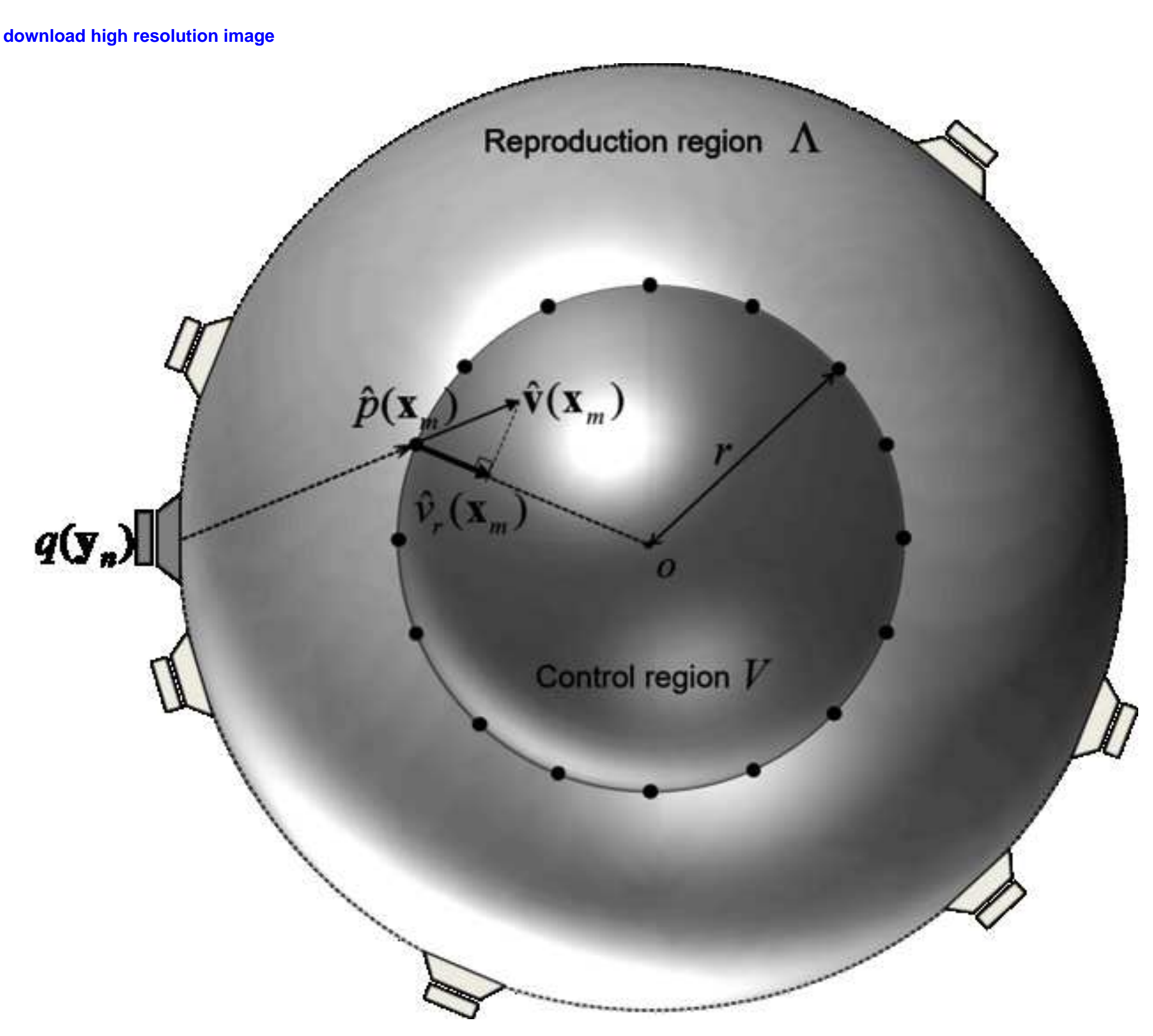



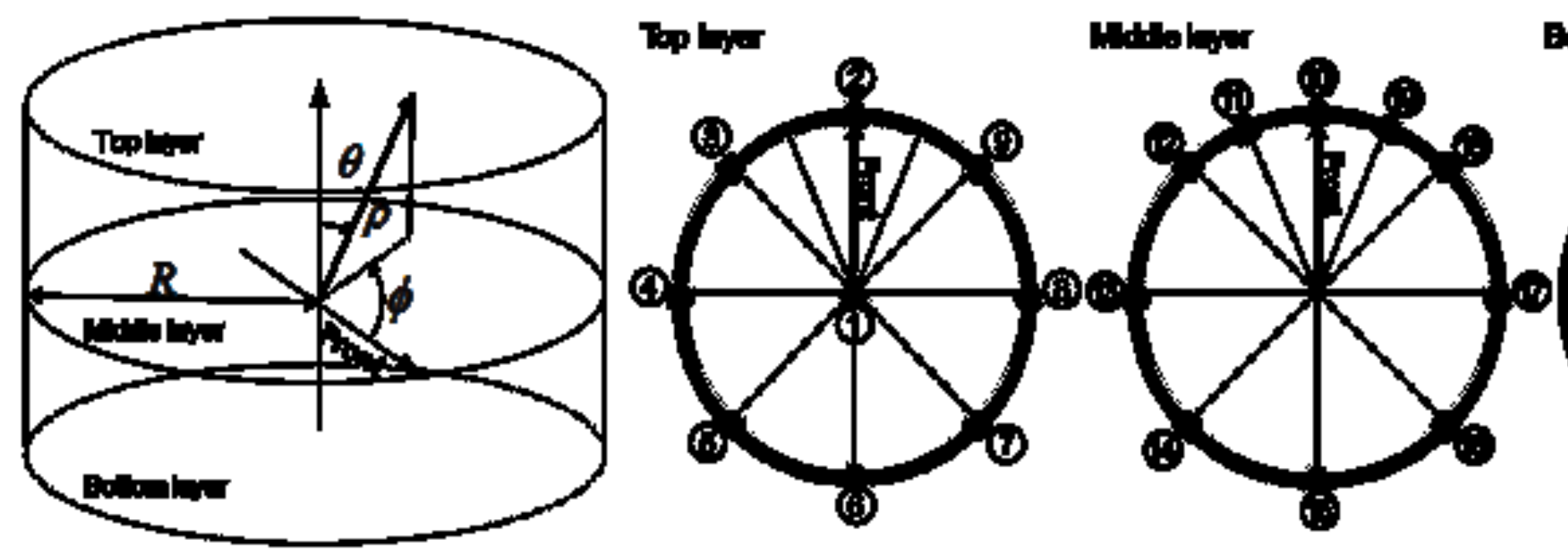

Bdrmb

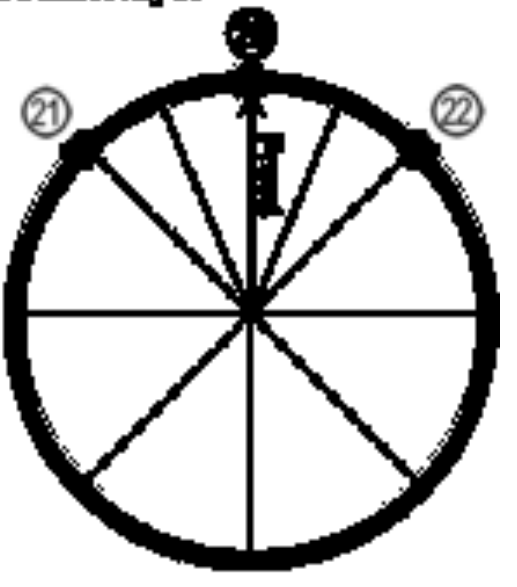




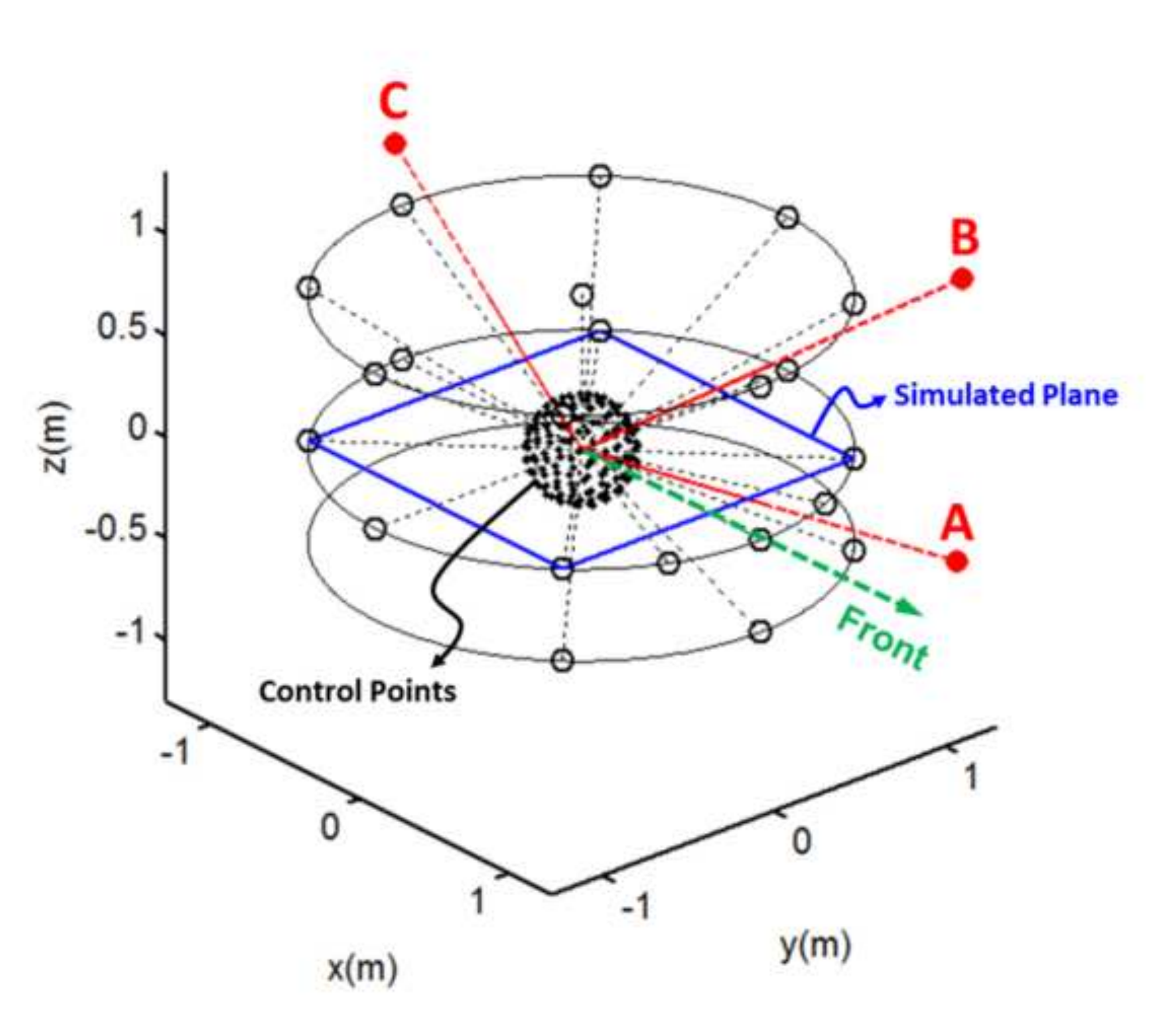

.

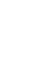

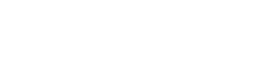

$$
n^{2}
$$

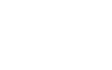

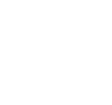

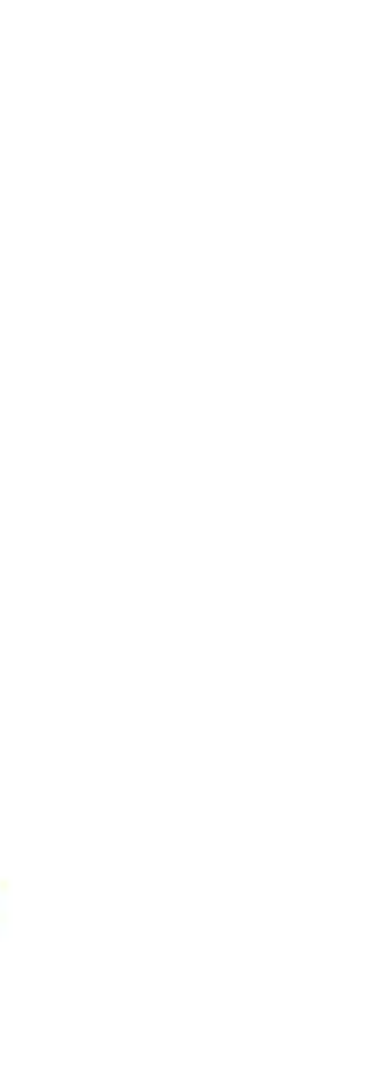
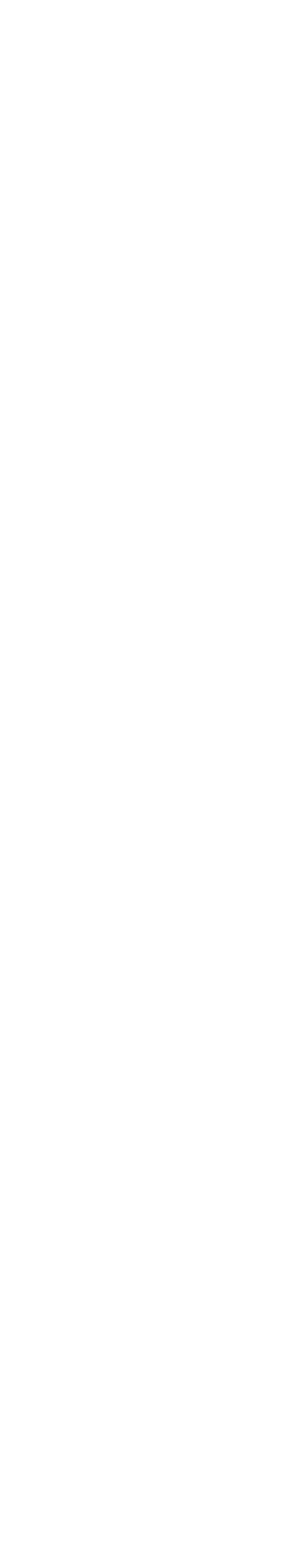
Click here to download high resolution image

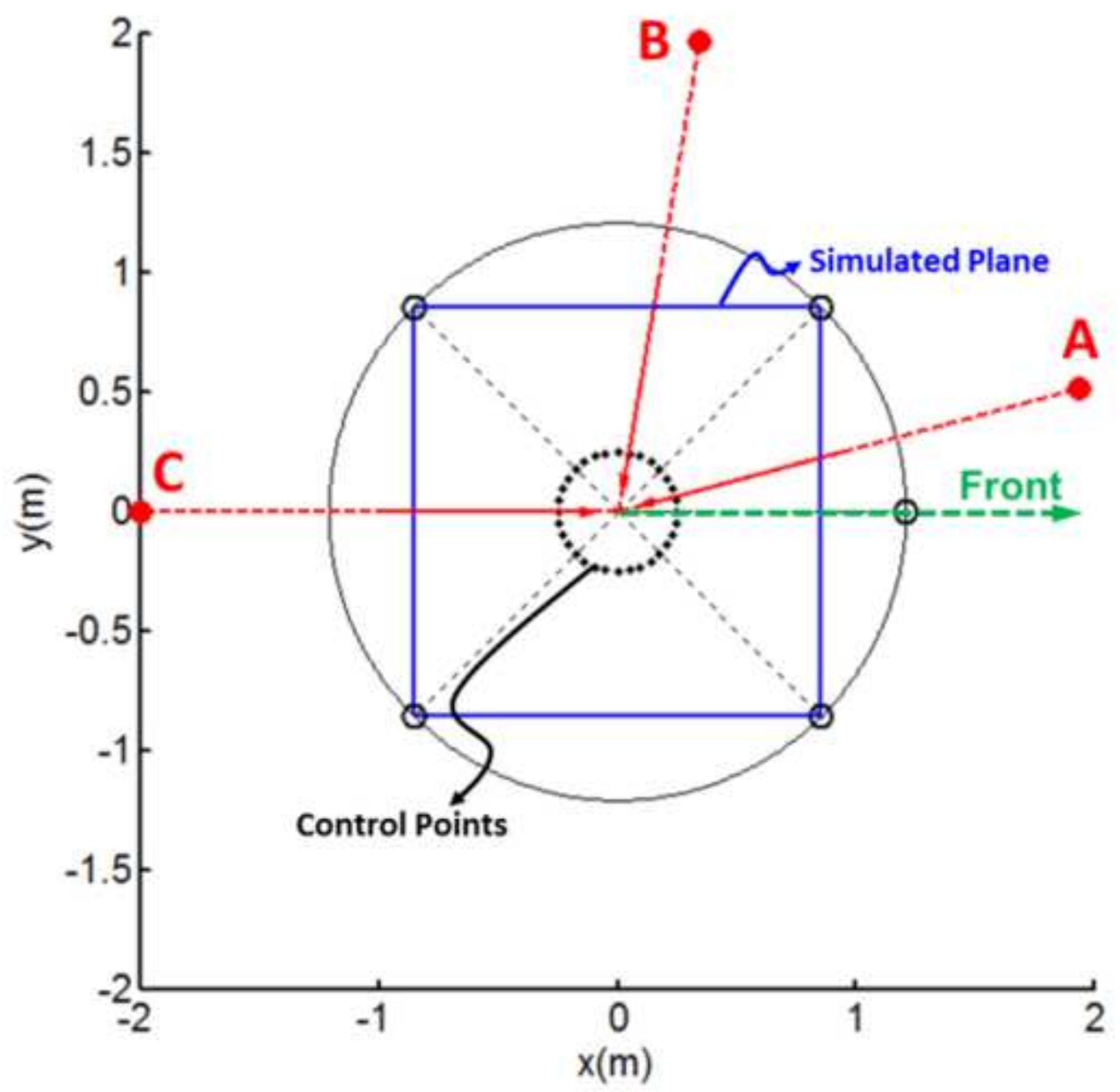


Click here to download high resolution image
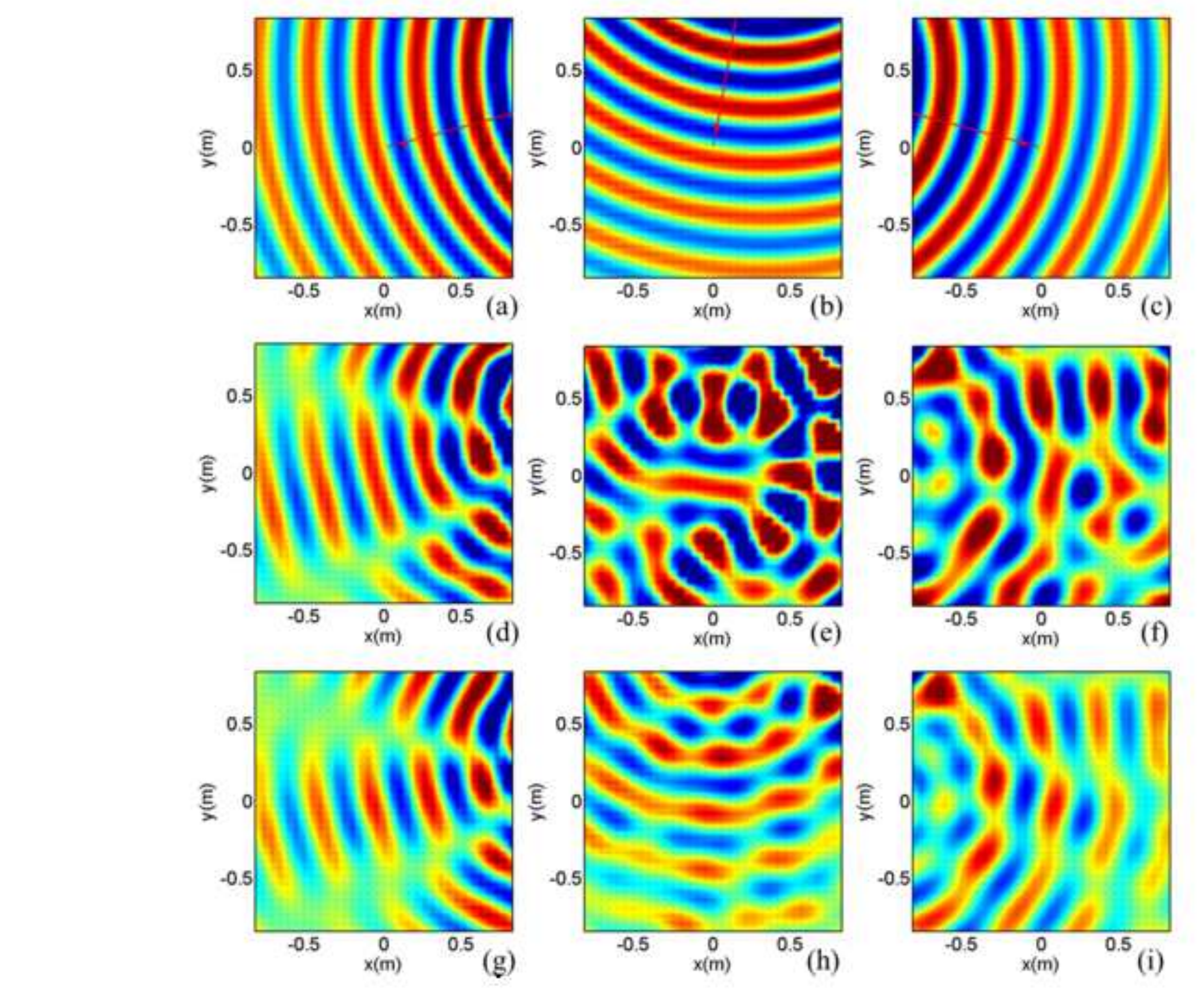

.

$$
\text { (n) }
$$$$
\text { . }
$$

$$
\text { " }
$$

$$
\text { - }
$$$$
\text { - }
$$

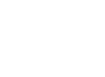

$$
\text { - }
$$

$$
\text { . }
$$

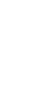

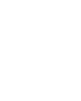



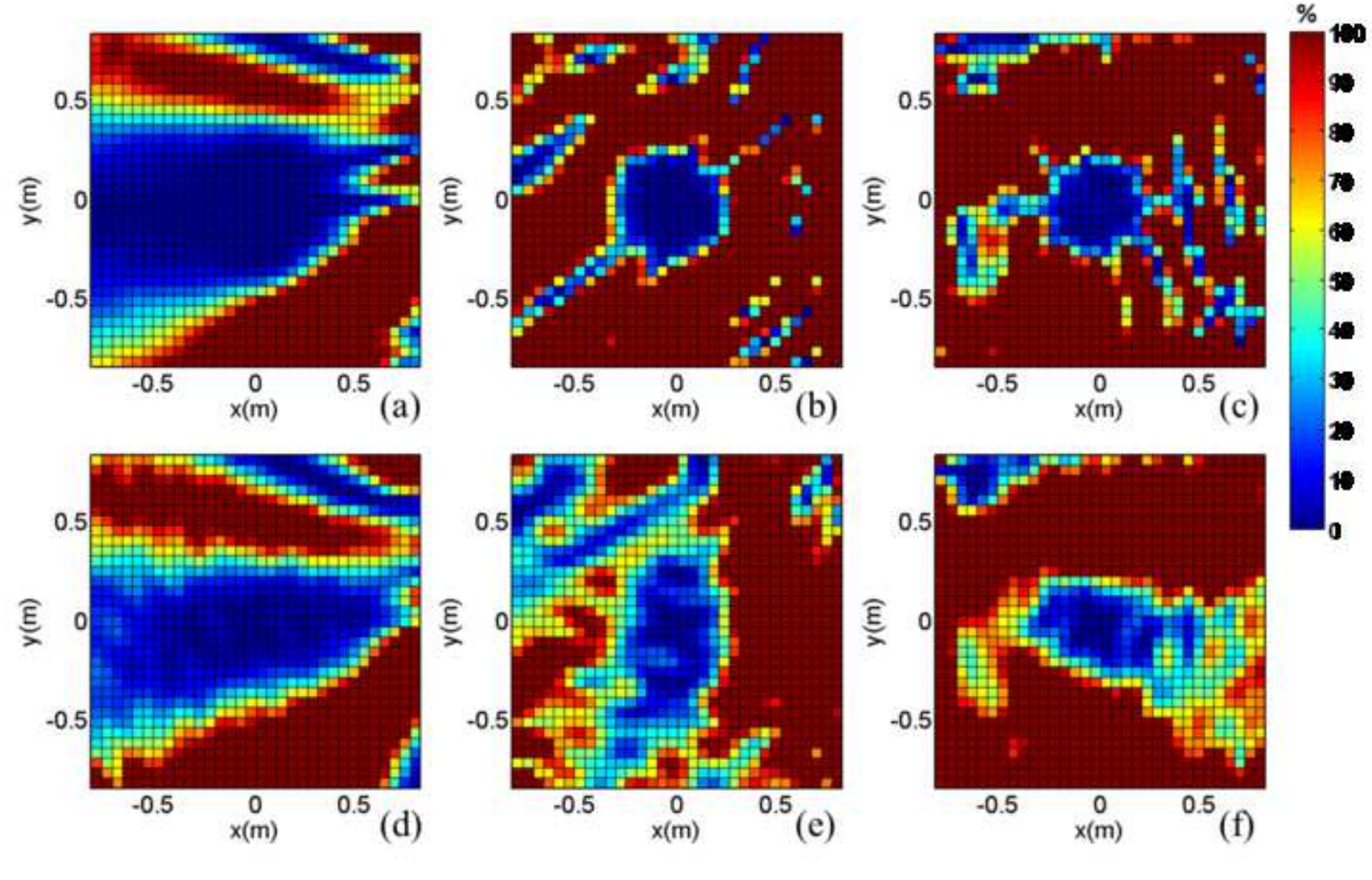

(2)

F

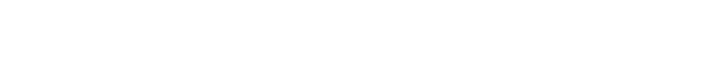$$
\text { (20) }
$$

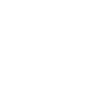

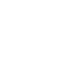

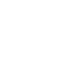

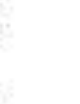



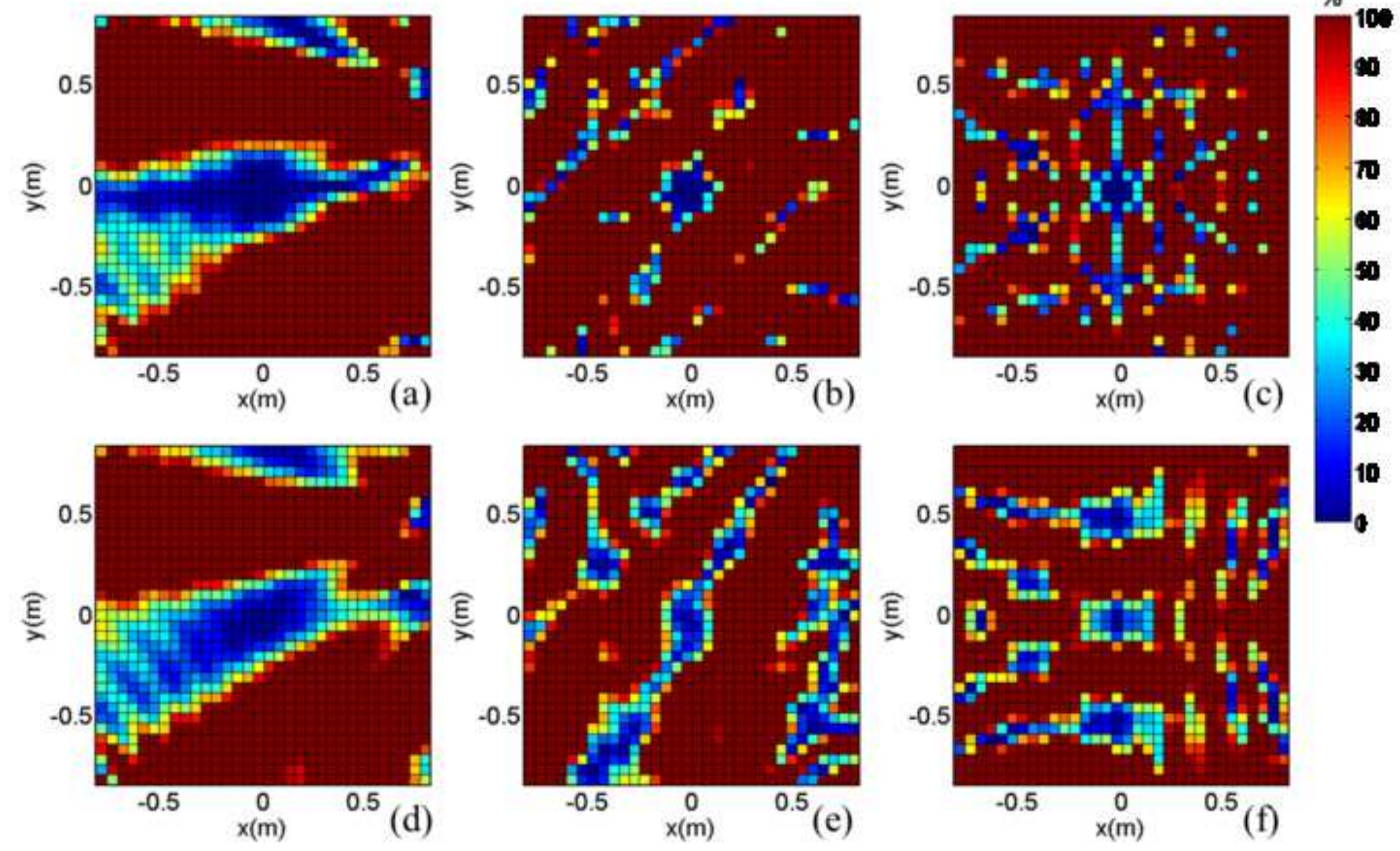

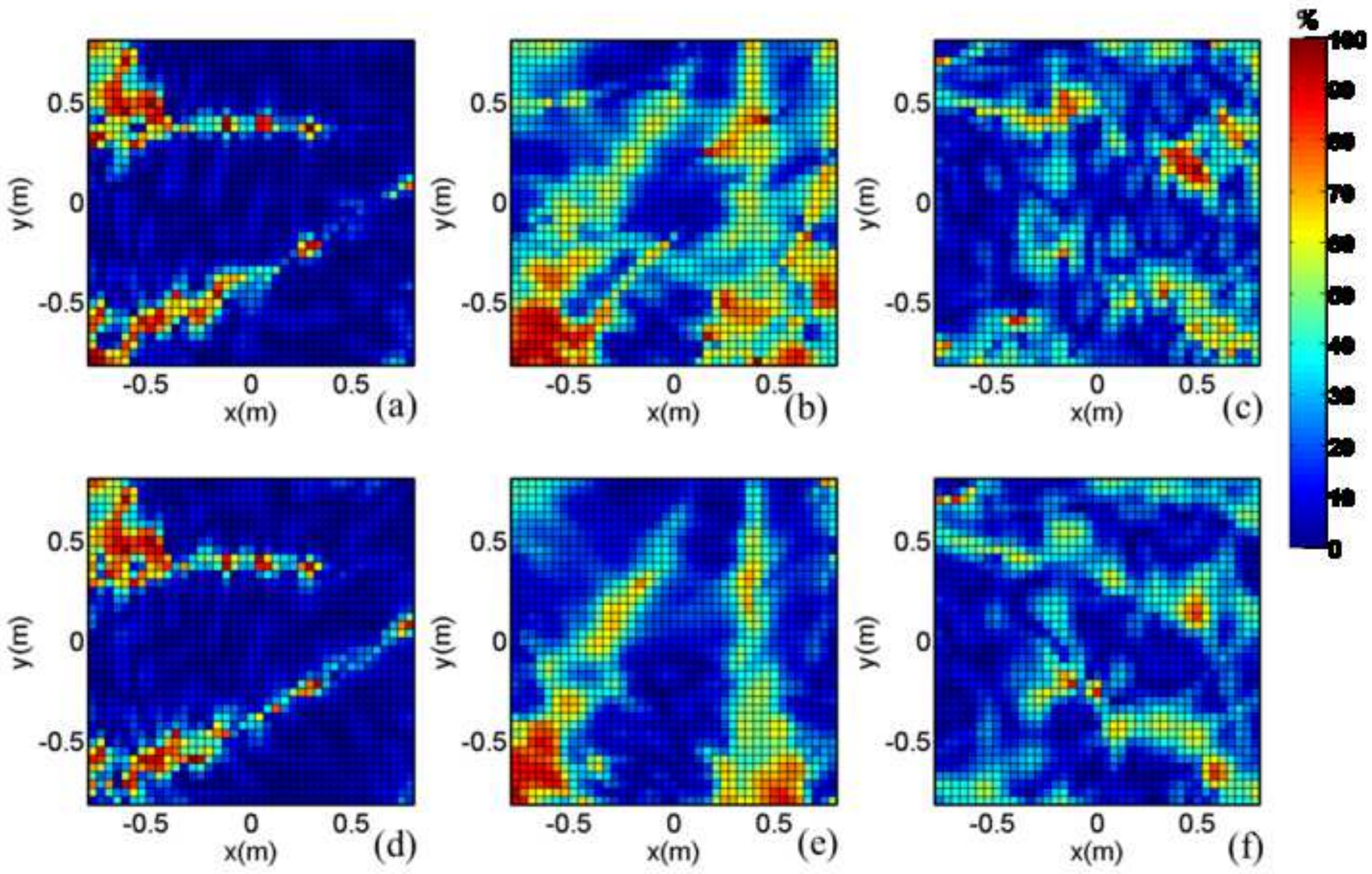

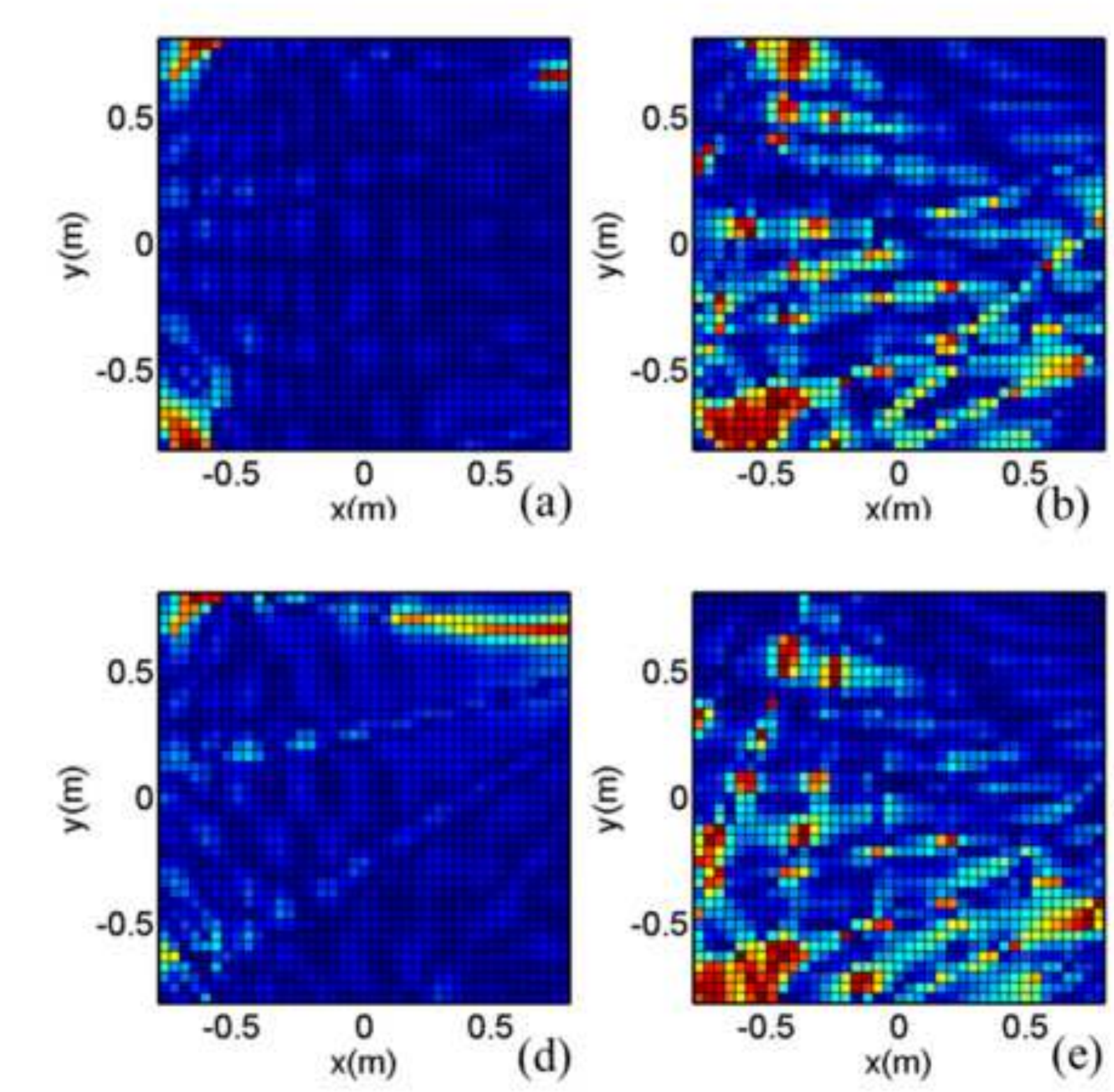

c.

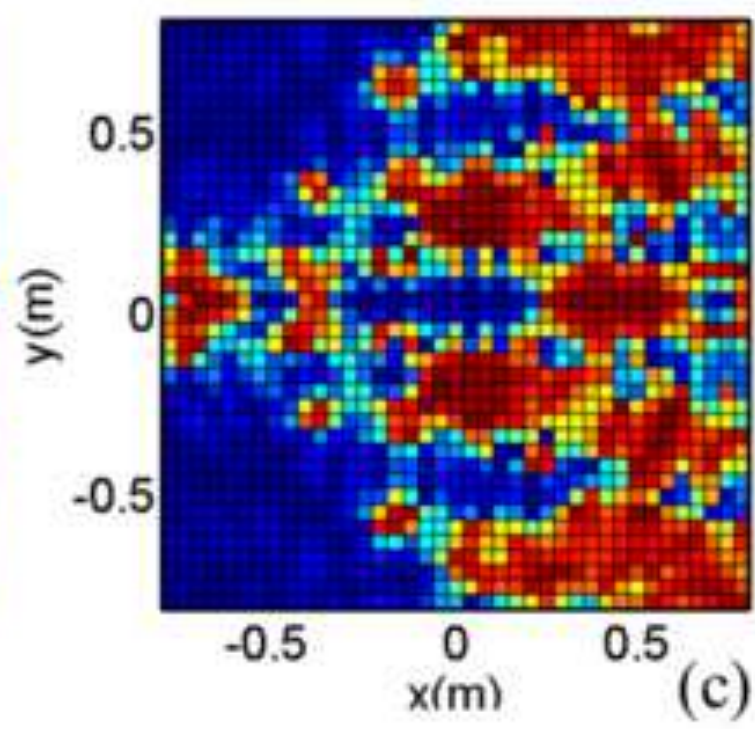

100

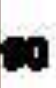

en

Iit

$\rightarrow$

$m$

1

(c)

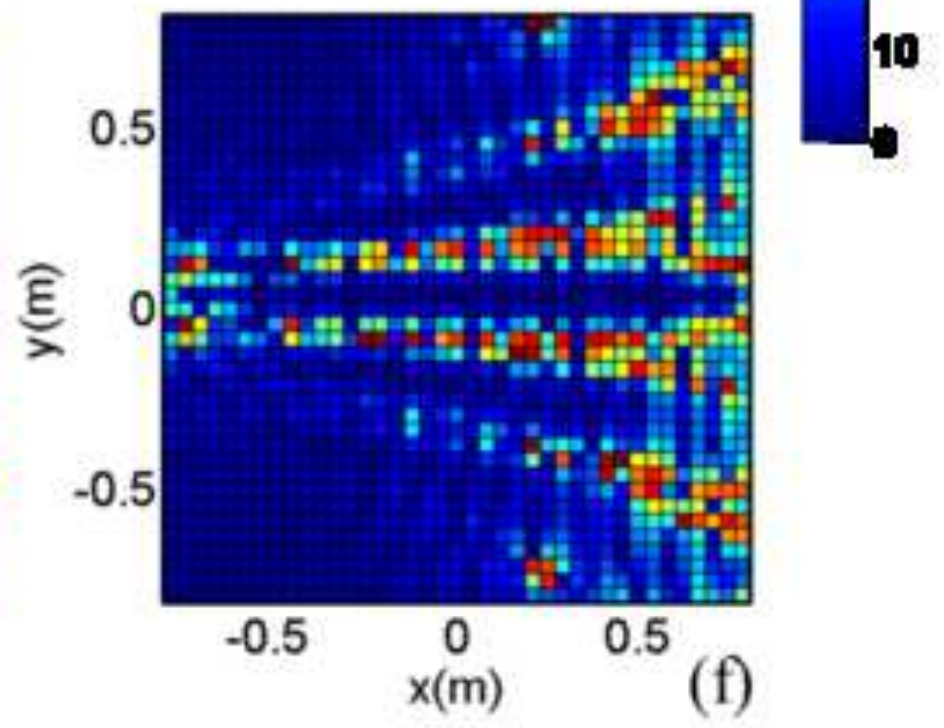



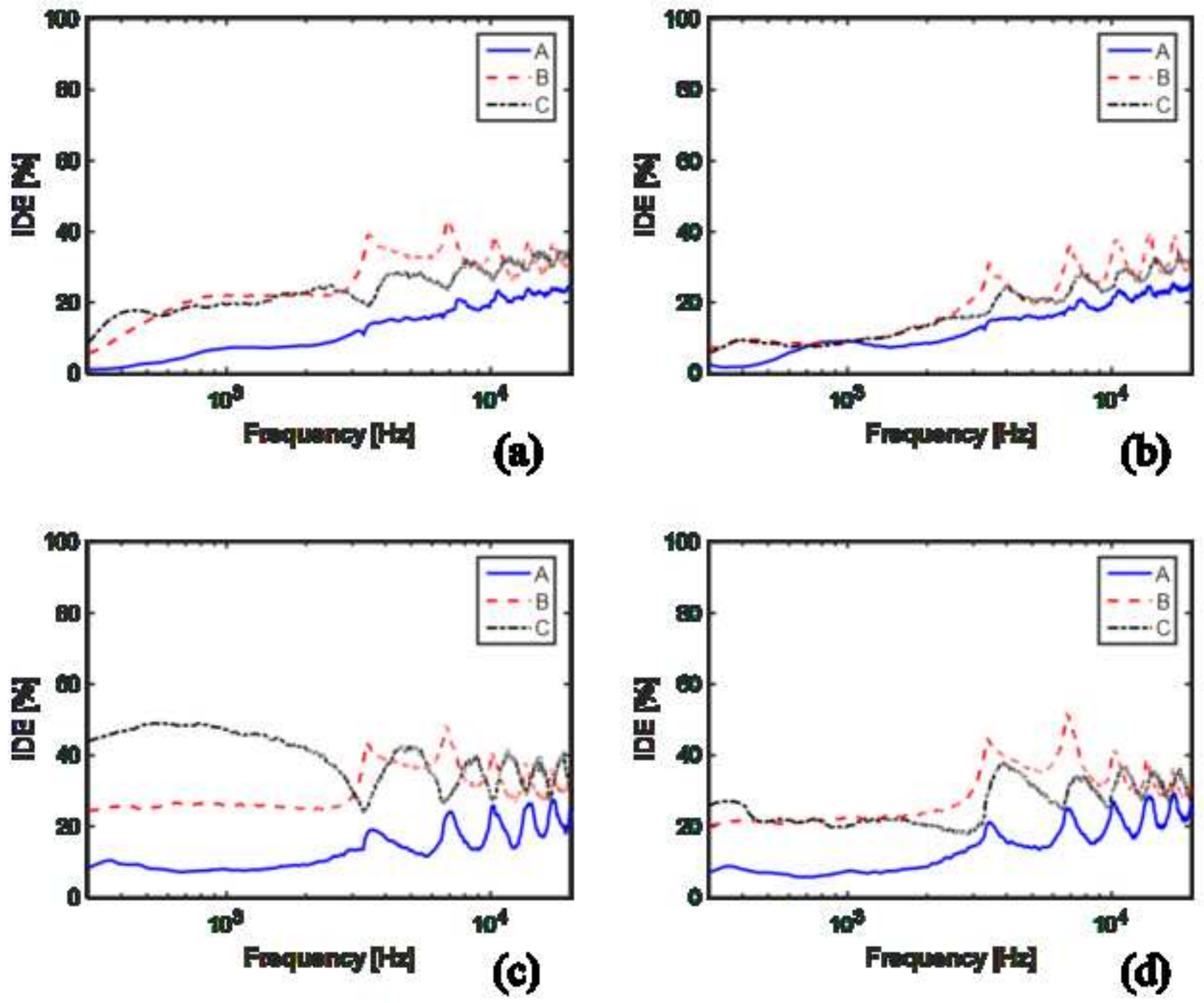

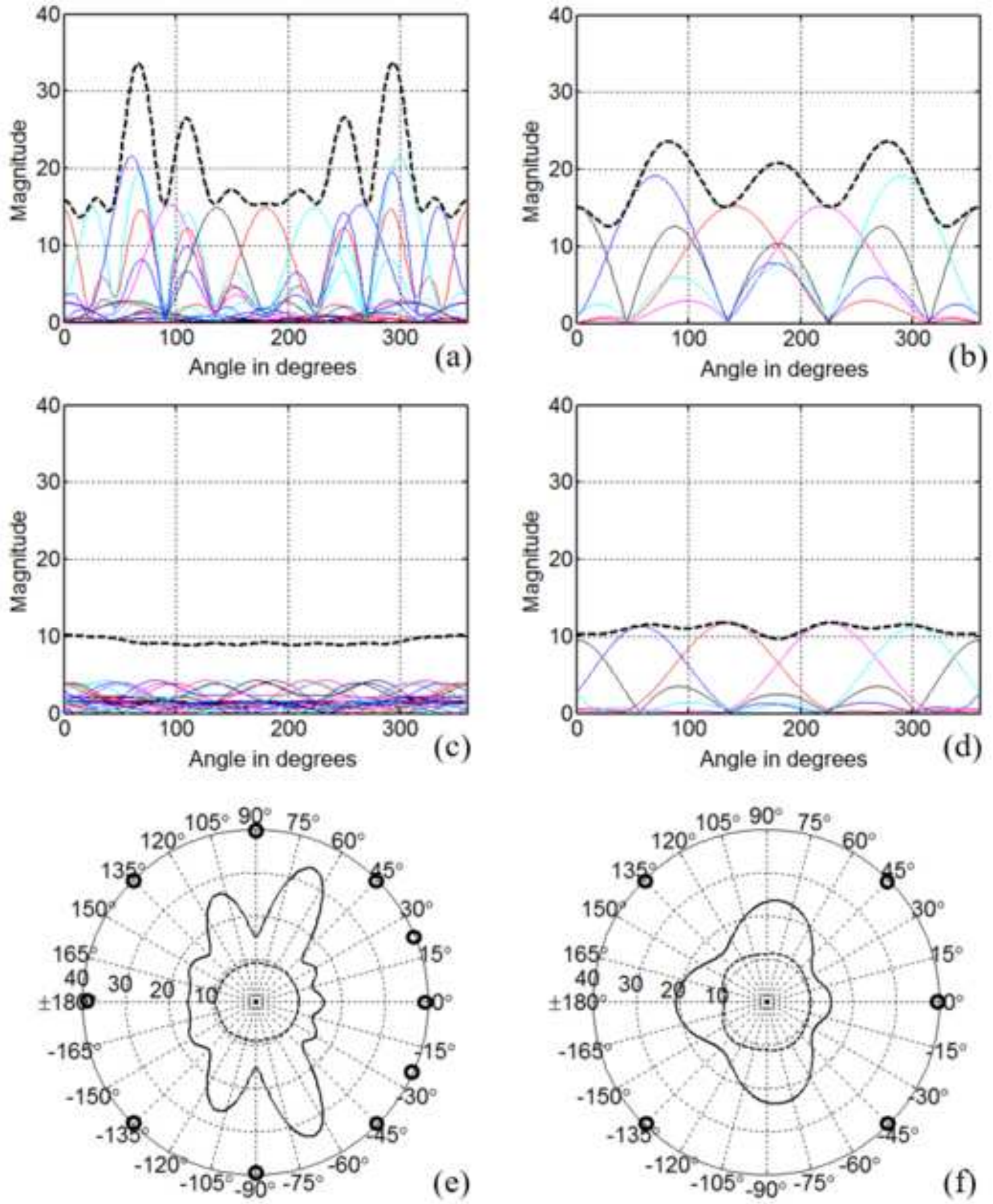

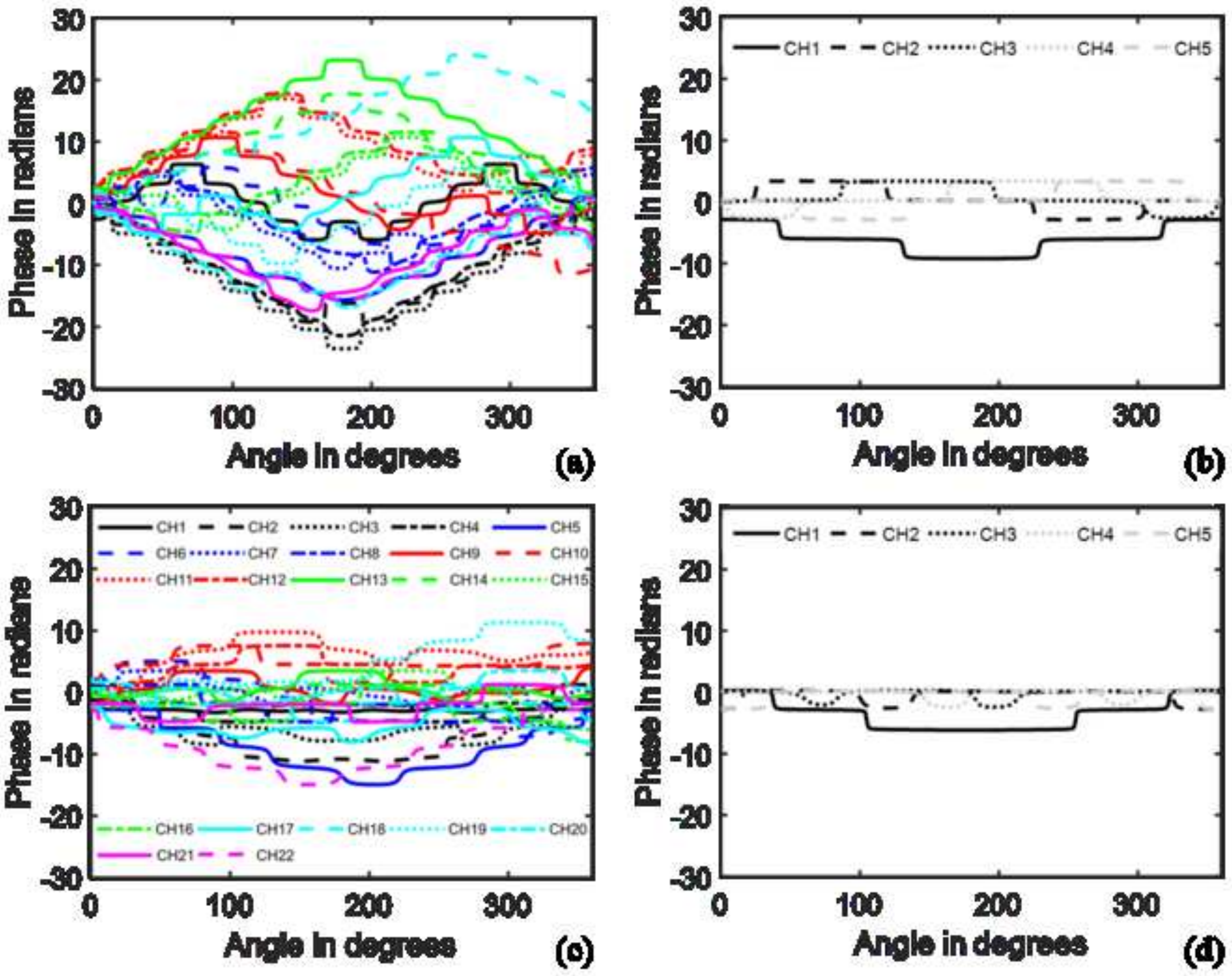

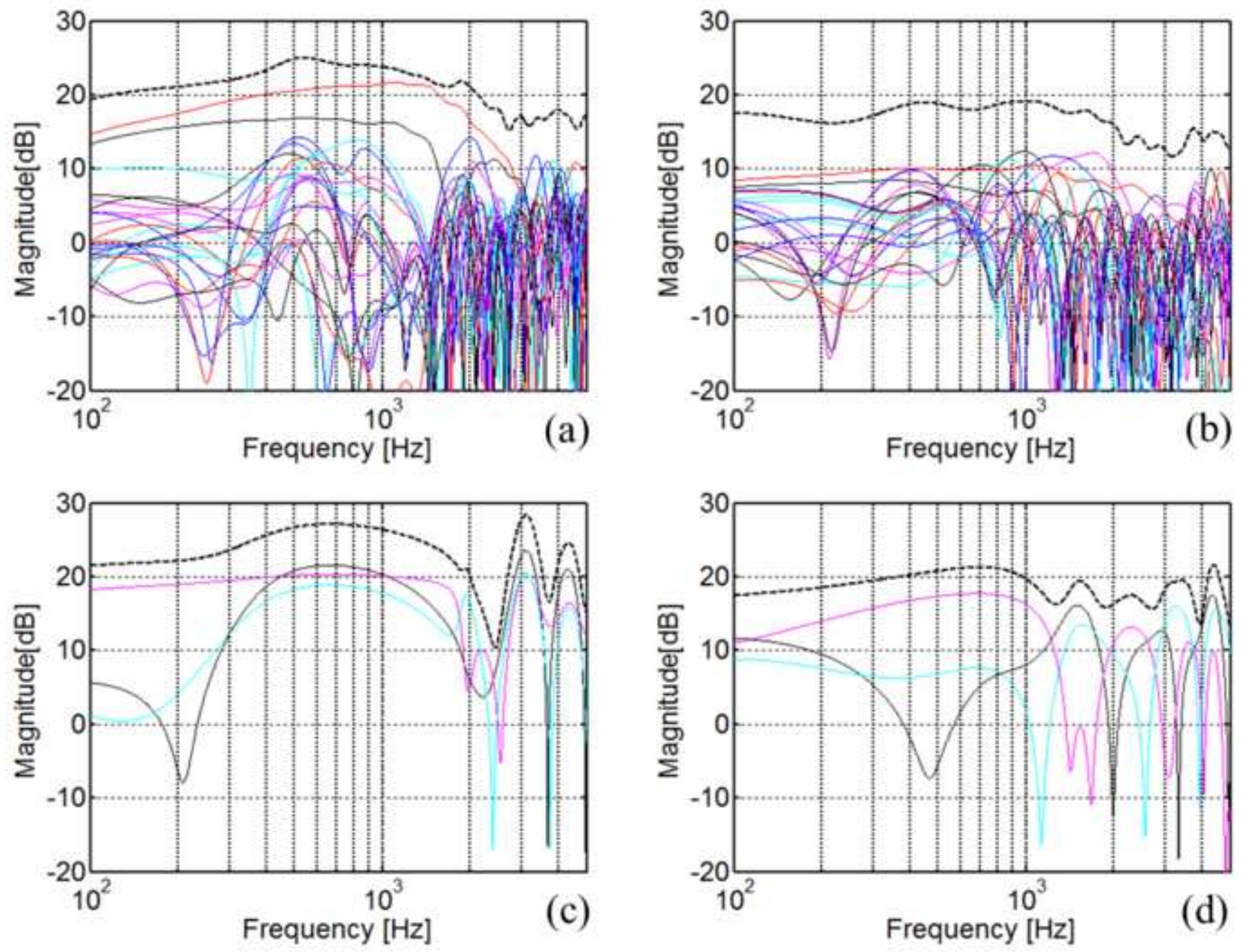

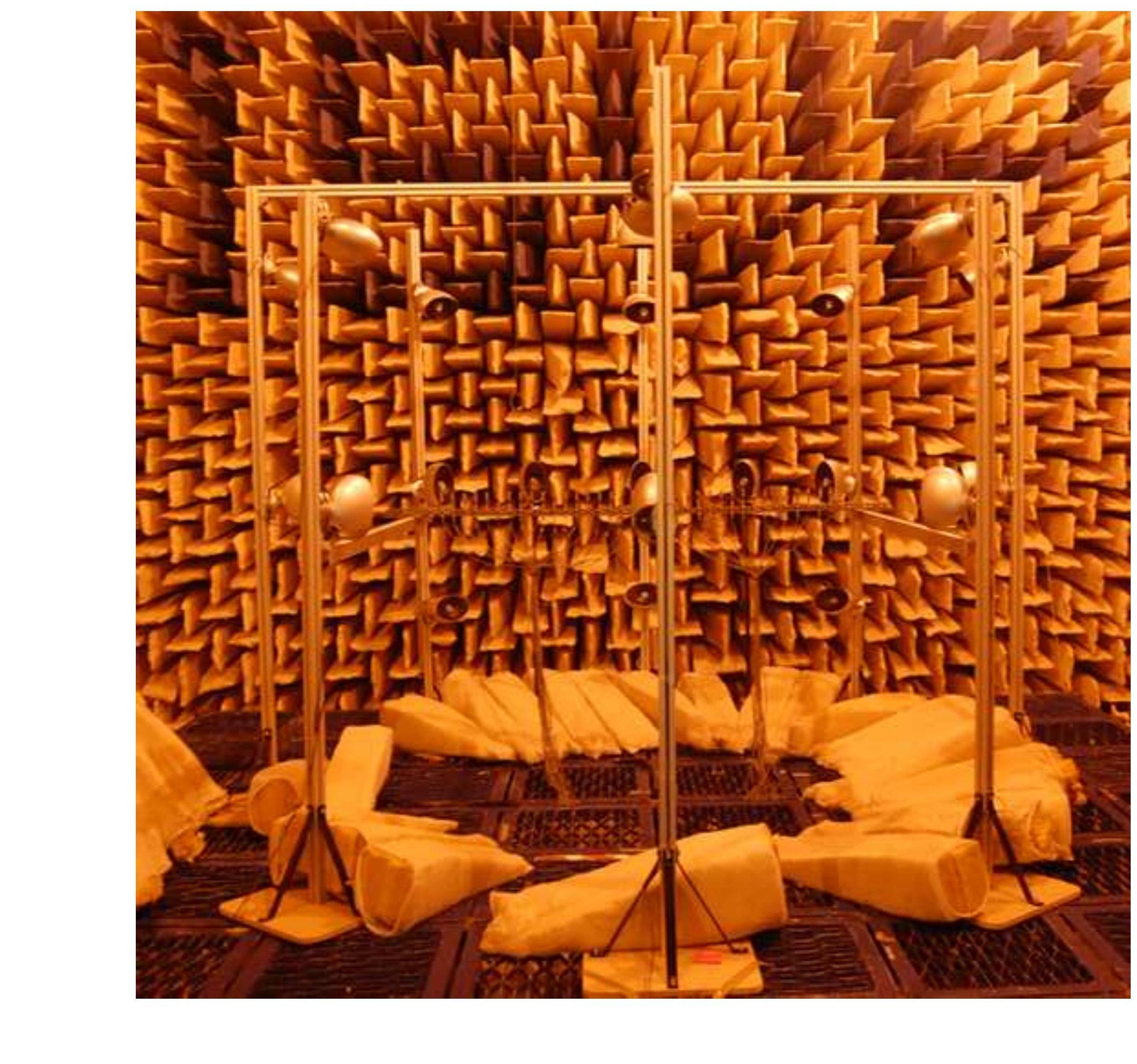
Click here to download high resolution image

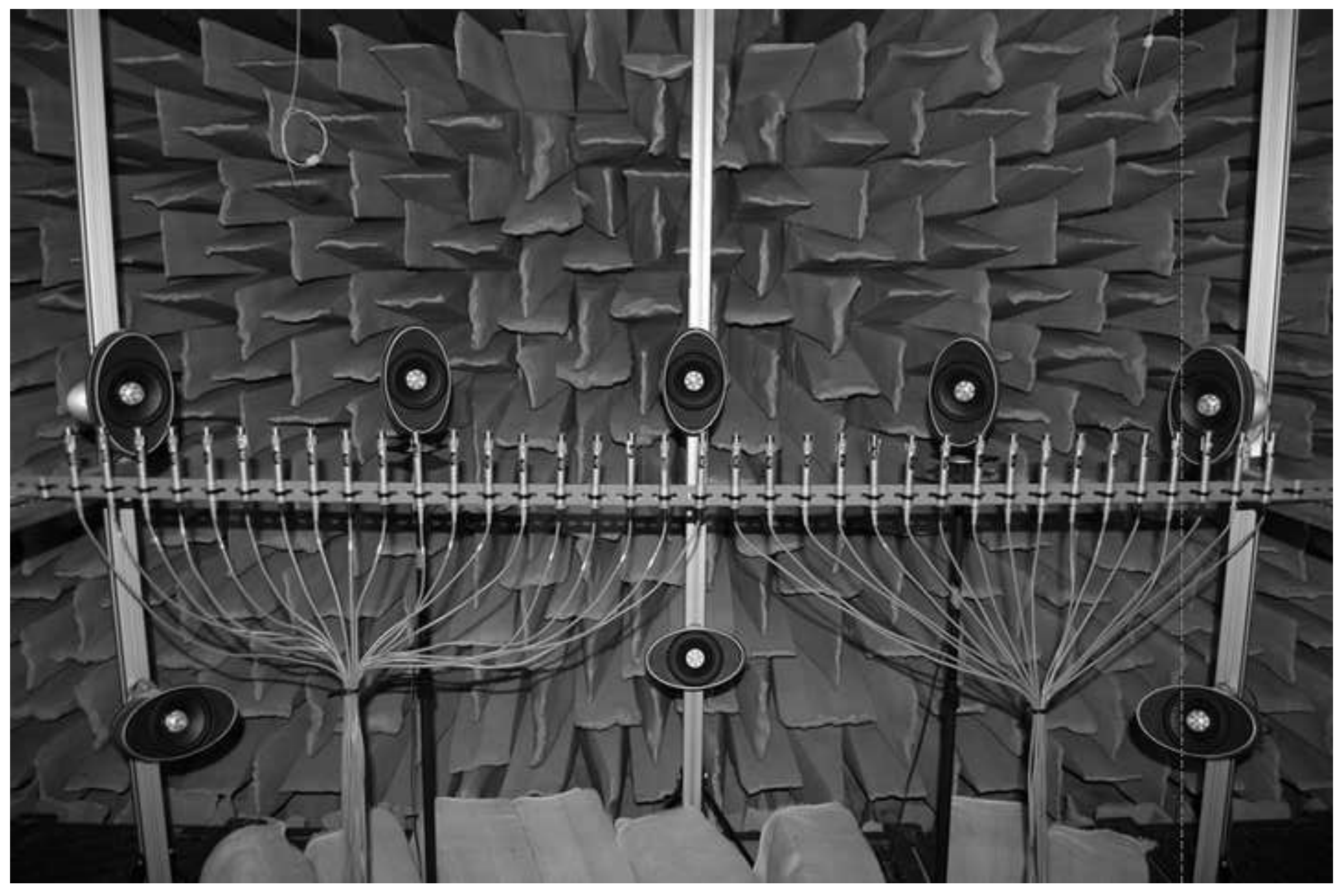



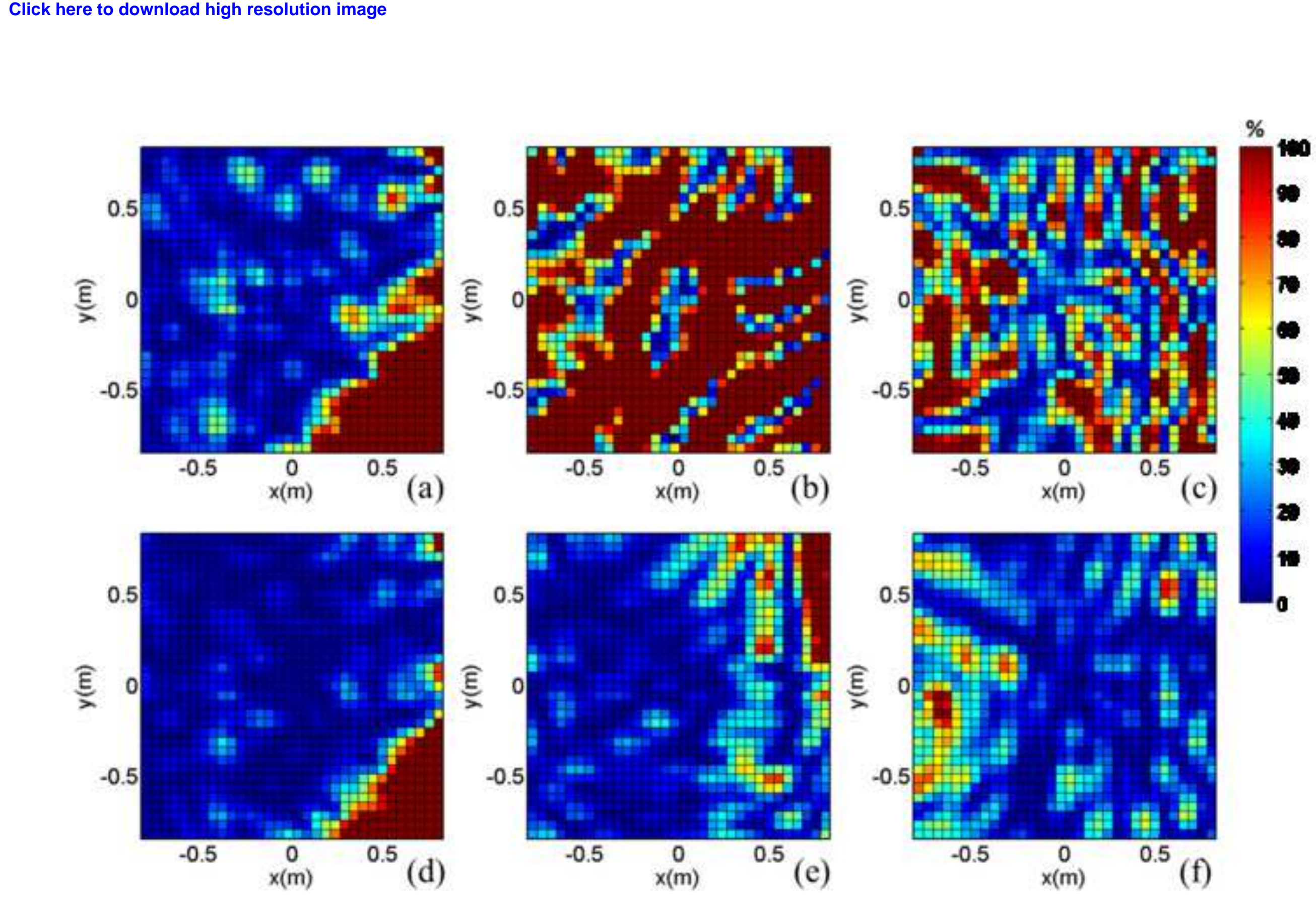

11.

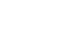

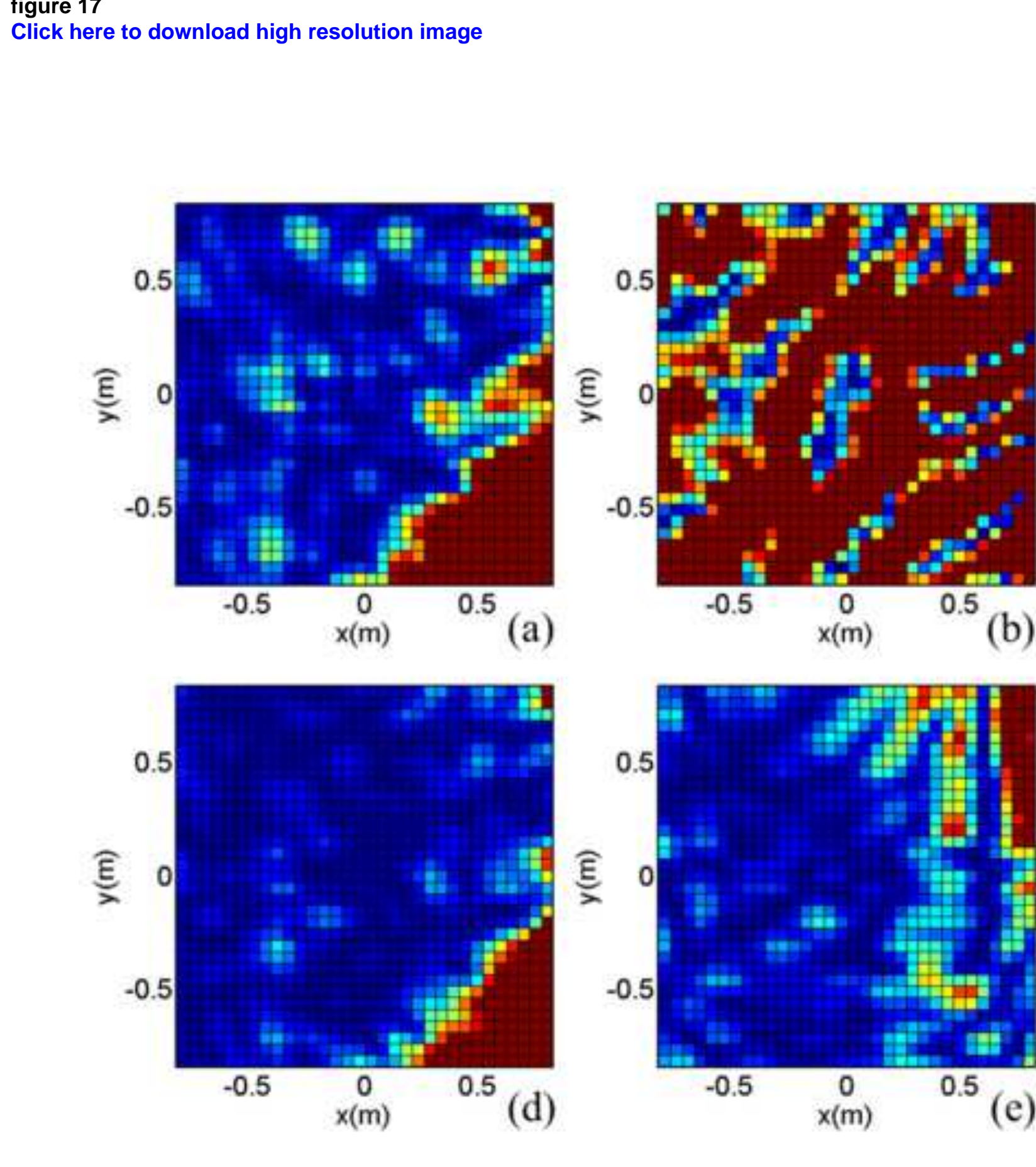


Click here to download high resolution image
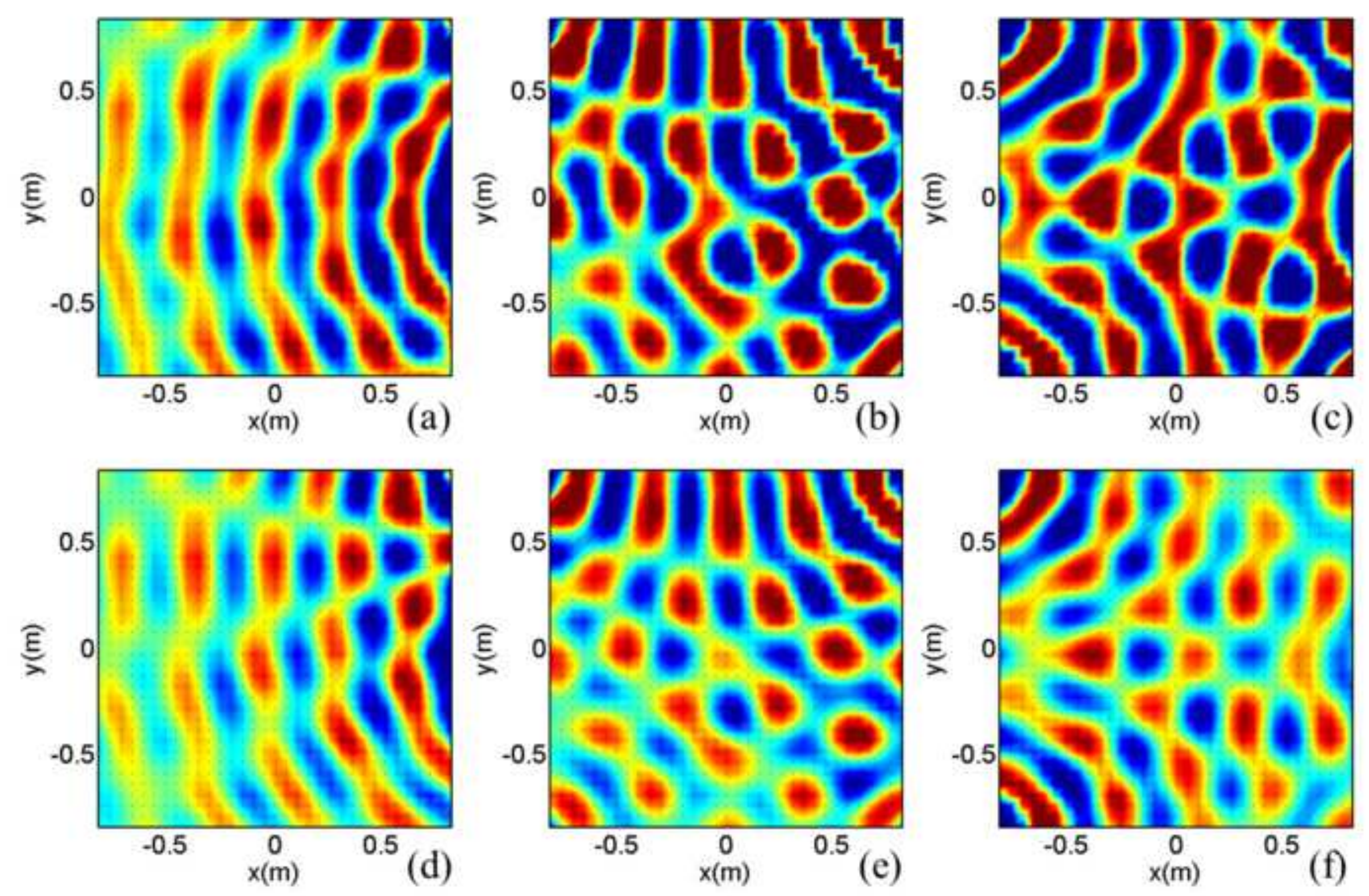$$
\text { (f) }
$$ 

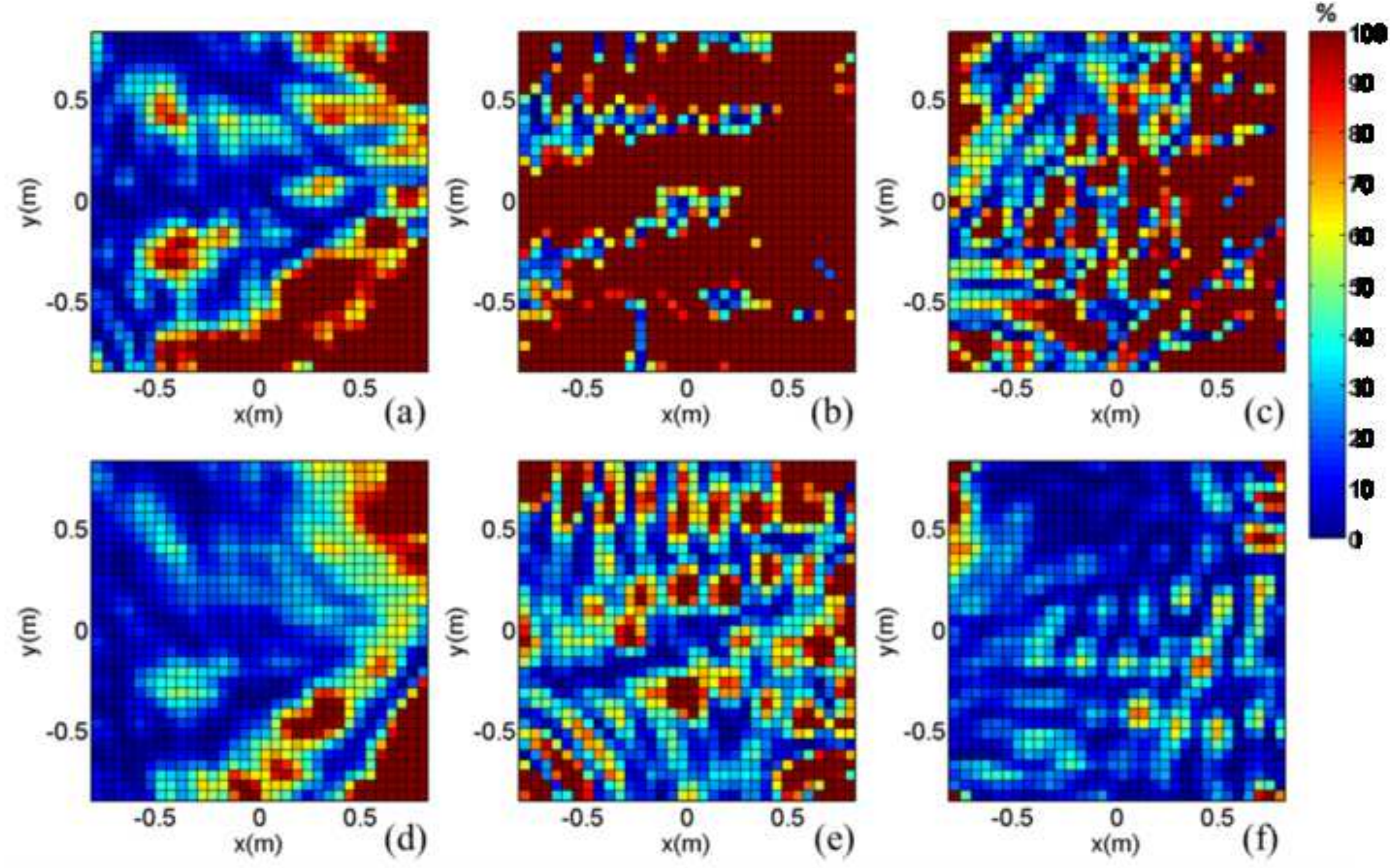

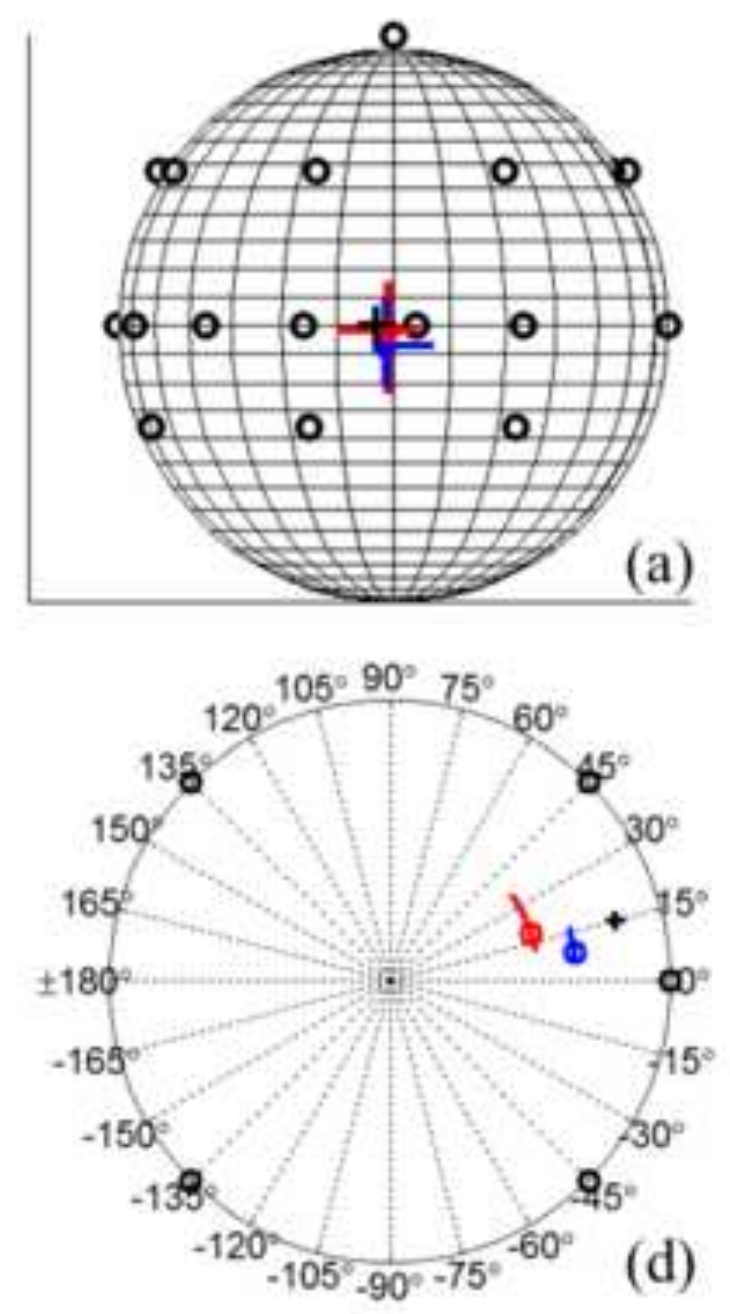

(d)
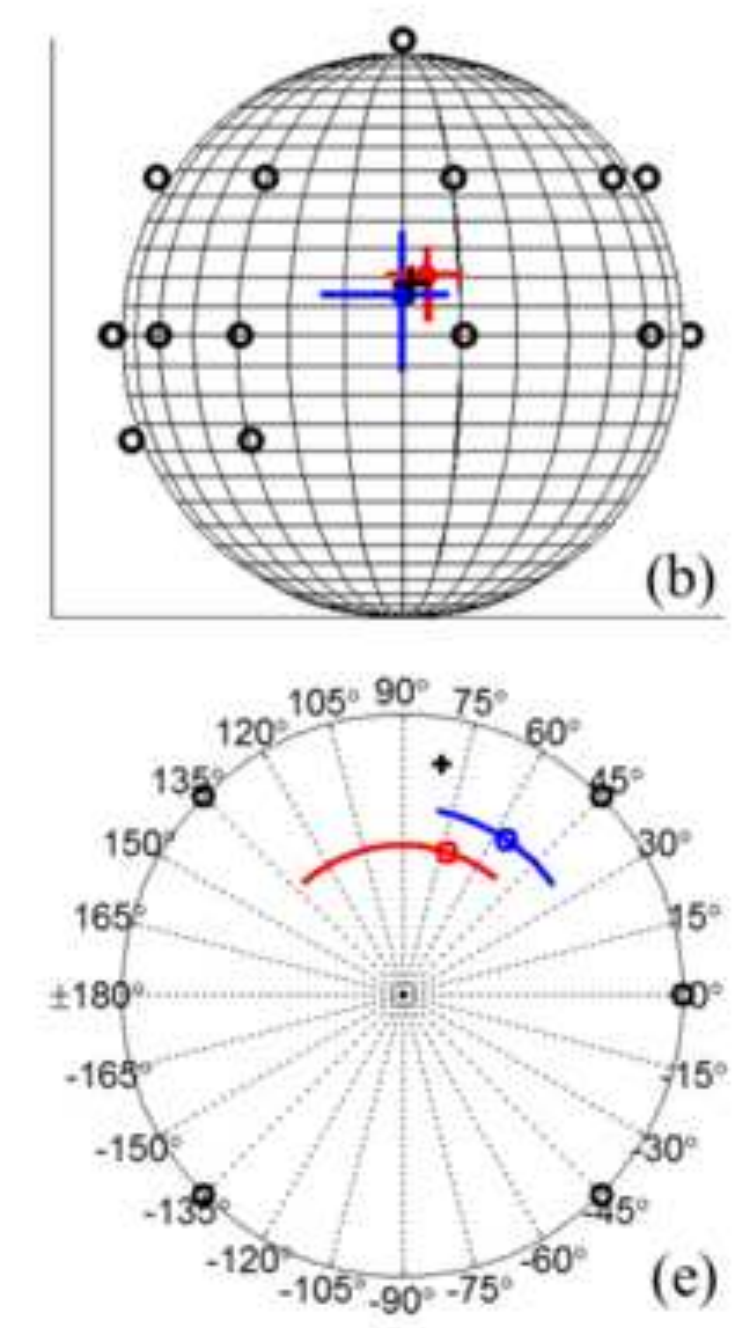

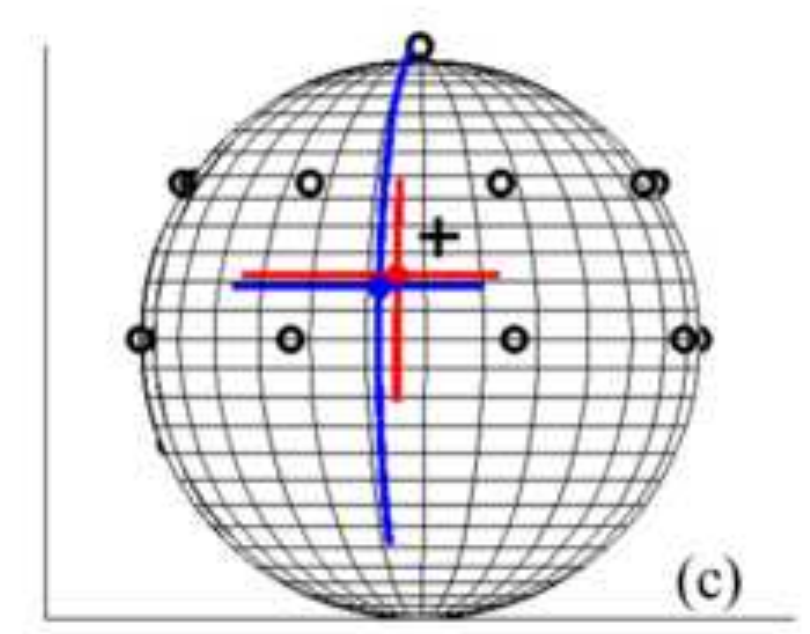

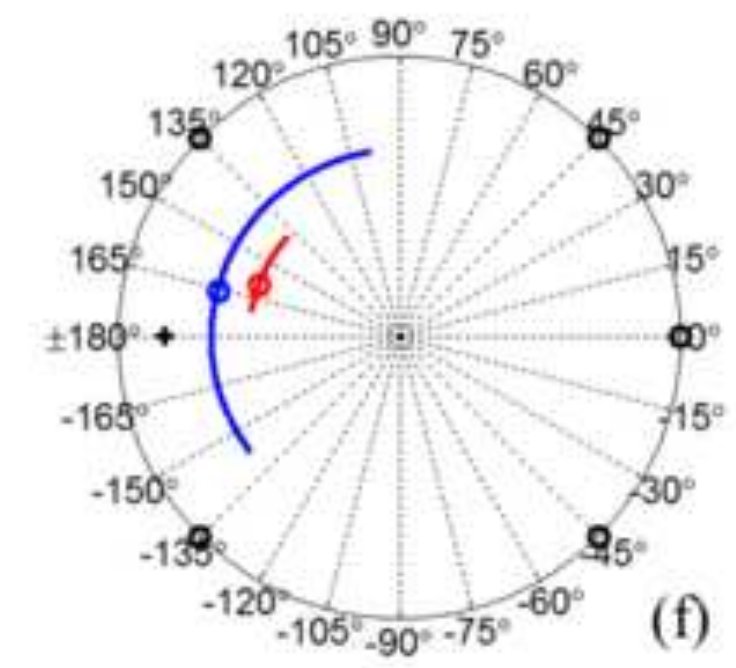

(b)

.

)

